\title{
Neutrino Anarchy and Renormalization Group Evolution
}

\author{
Vedran Brdar $^{1}$ * $^{*}$ Matthias König ${ }^{1 \dagger}$ and Joachim Kopp ${ }^{17}$ \\ ${ }^{1}$ PRISMA Cluster of Excellence and Mainz Institute for Theoretical Physics, \\ Johannes Gutenberg-Universität Mainz, 55099 Mainz, Germany
}

(Dated: September 26, 2018)

\begin{abstract}
The observed pattern of neutrino mixing angles is in good agreement with the hypothesis of neutrino anarchy, which posits that Nature has chosen the entries of the leptonic mixing matrix at random. In this paper we investigate how stable this conclusion is under renormalization group effects. Working in the simplest type-I seesaw model and two variants of the inverse seesaw model we study how the statistical distributions of the neutrino mixing parameters evolve between the Grand Unification scale and the electroweak scale. Especially in the inverse seesaw case we find significant distortions: mixing angles tend to be smaller after RG running, and the Dirac CP phase tends to be closer to zero. The $p$-value describing the compatibility between the observed mixing angles and the anarchy hypothesis increases by $10-20 \%$. This illustrates that RG effects are highly relevant for quantitative studies of the anarchy scenario.
\end{abstract}

\section{INTRODUCTION}

The flavor structure of leptons in the Standard Model (SM) is experimentally well established, but our theoretical understanding of its origin is very poor. The simplest postulate is that there is no particular structure to the neutrino mass matrix, but that its elements are randomly chosen $\mathcal{O}(1)$ parameters. This scenario is called neutrino anarchy [1, 2]. While one may view the idea of nature choosing model parameters at random with some reservation, it does not seem so unnatural given that nature also chooses the outcome of quantum mechanical measurements at random. Moreover, it can be considered a valid low-energy description if the underlying UV-complete theory is sufficiently complicated [2].

Once we accept the idea of anarchy, it can be used to derive meaningful predictions for the values of the neutrino mixing parameters. In particular, as shown in [2], the requirement that different elements of the mass matrices are statistically independent and that their distributions are basis-independent leads uniquely to the conclusion that the lepton mixing matrix $U_{\text {PMNS }}$ must have a flat distribution in the Haar measure of the $U(3)$ group. This allows one to compute $p$ values for the experimentally measured mixing parameters $[2,5]$. The original neutrino anarchy scenario [1] has been criticized in [4] on the grounds that it cannot be formulated unambiguously if mass matrices that only differ in their unphysical phases are considered identical. This viewpoint is valid if one assumes that nature does not "know" about the unphysical phases at all, i.e. that they do not appear as hidden variables in the theory. It becomes invalid if we accept the unphysical phases as equipollent with the physical ones, for instance on the grounds that they might have physical significance in an underlying UV-complete theory.

Of course the idea of anarchy is not restricted to the neutrino mass models in which it was originally proposed, but has also been applied for instance to scenarios with sterile neutrinos [6, 7], models with extra dimensions [8, and in the context of grand unified theories (GUTs) 9 11. Moreover, the idea that physical parameters are drawn from a statistical distribution has received some attention in the context of the string landscape, see for instance [12, 13].

\footnotetext{
* Email: vbrdar@uni-mainz.de

† Email: m.koenig@uni-mainz.de

‡ Email: jkopp@uni-mainz.de
} 
In this paper we investigate how stable the predictions of neutrino anarchy are under the effects of renormalization group ( $R G$ ) running. This is an important question given that we do not know how and at what scale the random selection of model parameters happens. It could be a feature of a grand unified theory, of quantum gravity, or it could be an outcome of the dynamics at the end of inflation. We will show that in the simplest type-I seesaw scenario, which augments the SM by three heavy right-handed neutrinos, mixing angles derived from anarchical mass matrices at a high energy scale can differ from those at experimentally testable scales by several degrees. The probability of the neutrino oscillation parameters being at their experimentally observed values under the anarchy hypothesis increases by $\sim 7 \%$ because of this. We show that this result does not depend on the statistical test we choose to make the comparison. We also investigate how our results change in extended neutrino mass models. In particular, we show that renormalization group effects become larger in inverse seesaw models.

The plan of the paper is as follows: In section II, we will review neutrino anarchy and its predictions without including renormalization group effects. Comparing these predictions to data quantitatively, we will show that results are robust with respect to the choice of statistical test. Section III contains our main results: we will investigate how the predictions of neutrino anarchy are modified when renormalization group running from the GUT breaking scale $M_{\mathrm{GUT}} \sim 10^{16} \mathrm{GeV}$ to the electroweak scale is taken into account. We will do this for the type-I seesaw scenario (section III.1), for the phenomenological inverse seesaw model (section III.2), and for a particular realization of the inverse seesaw model involving a gauged $U(1)_{B-L}$ symmetry (section III.3). Besides looking at the mixing angles and complex phases, we will also study the predictions that neutrino anarchy makes for the effective neutrino mass parameter probed in searches for neutrinoless double beta $(0 \nu 2 \beta)$ decay. We will present our conclusions in section IV. In the appendices, we will offer details on the renormalization group equations we employ. In particular for the inverse seesaw model with $U(1)_{B-L}$ symmetry, these equations have, to the best of our knowledge, not been presented before.

\section{NEUTRINO ANARCHY}

\section{II.1. Statistical tests}

We consider first the type-I seesaw scenario, where the SM is extended by adding three righthanded singlet fermions $N_{R}^{\alpha}$. The relevant terms in the Lagrangian are

$$
\mathcal{L}_{\text {type-I }} \supset-Y_{e}^{\alpha \beta} \overline{e_{R}^{\alpha}} H^{\dagger} L^{\beta}-Y_{\nu}^{\alpha \beta} \overline{N_{R}^{\alpha}} \tilde{H}^{\dagger} L^{\beta}-\frac{1}{2} M^{\alpha \beta} \overline{N^{\alpha}}\left(N^{\beta}\right)^{c}+\text { h.c. },
$$

where $L^{\alpha}=\left(\nu_{L}^{\alpha}, e_{L}^{\alpha}\right)$ are the left-handed lepton doublets $(\alpha=e, \mu, \tau), e_{R}^{\alpha}$ are the right-handed charged lepton fields, $H$ is the SM Higgs doublet, and $\tilde{H}=i \sigma^{2} H^{*}$ is the charge conjugate Higgs field. The Yukawa matrices $Y_{e}$ and $Y_{\nu}$ are general complex $3 \times 3$ matrices, while the Majorana mass matrix $M$ for the right-handed neutrinos is a complex symmetric $3 \times 3$ matrix. After electroweak symmetry breaking, the Higgs field acquires a vacuum expectation value (vev) $\langle H\rangle=(0, v / \sqrt{2})$.

If the eigenvalues of $M$ are much larger than those of $m_{D} \equiv Y_{\nu} v / \sqrt{2}$, the effective neutrino mass term at low energies becomes

$$
\mathcal{L}_{m, \text { type-I }} \supset-\frac{1}{2} m_{\nu}^{\alpha \beta} \overline{\left(\nu_{L}^{\alpha}\right)^{c}} \nu_{L}^{\beta}+\text { h.c. },
$$

with

$$
m_{\nu}=-v\left(\frac{v}{2} Y_{\nu}^{T} M^{-1} Y_{\nu}+\mathcal{O}\left(\frac{v^{3}}{M^{3}}\right)\right)
$$


The charged lepton mass matrix $m_{e} \equiv Y_{e} v / \sqrt{2}$ is diagonalized by a transformation $e_{L}^{\alpha} \rightarrow V_{e}^{\alpha \beta} e_{L}^{\beta}$, $e_{R}^{\alpha} \rightarrow V_{e}^{\prime \alpha \beta} e_{R}^{\beta}$, and the neutrino mass matrix $m_{\nu}$ is diagonalized by a transformation $\nu_{L}^{\alpha} \rightarrow V_{\nu}^{\alpha \beta} \nu_{L}^{\beta}$. The leptonic mixing matrix $U_{\mathrm{PMNS}}$ is then given by $U_{\mathrm{PMNS}}=V_{e}^{\dagger} V_{\nu}$.

The anarchy hypothesis states that the entries of $m_{D}$ and $M$ are randomly and independently drawn from a statistical distribution. One usually imposes the additional requirement that this distribution is the same in any basis, i.e. it is invariant under transformations of the form $\nu_{L}^{\alpha} \rightarrow$ $U^{\alpha \beta} \nu^{\beta}, N^{\alpha} \rightarrow U^{\alpha \beta} N^{\beta}$, where $U$ and $U^{\prime}$ are unitary matrices. One can show [2] that basis independence implies that the parameters of $U_{\mathrm{PMNS}}$ have a flat distribution in the Haar measure [2]. If the $U(3)$ matrix $U_{\mathrm{PMNS}}$ is parameterized in terms of the three mixing angles $\theta_{12}, \theta_{13}, \theta_{23}$, the Dirac CP phase $\delta_{\mathrm{CP}}$, the two Majorana CP phases $\phi_{1}, \phi_{2}$, and the three unphysical phases $\delta_{e}, \delta_{\mu}$, $\delta_{\tau}$ (see for instance [14]), the Haar measure reads

$$
d U_{\mathrm{PMNS}}=d\left(\sin ^{2} \theta_{12}\right) d\left(\sin ^{2} \theta_{23}\right) d\left(\cos ^{4} \theta_{13}\right) d \delta_{\mathrm{CP}} d \phi_{1} d \phi_{2} d \delta_{e} d \delta_{\mu} d \delta_{\tau} .
$$

A flat distribution in the Haar measure thus means that the distributions of the parameters $\sin ^{2} \theta_{12}$, $\sin ^{2} \theta_{23}, \cos ^{4} \theta_{13}, \delta_{\mathrm{CP}}, \phi_{1}, \phi_{2}, \delta_{e}, \delta_{\mu}$, and $\delta_{\tau}$ are flat within their physically allowed ranges [15]. In order to obtain a Haar-flat leptonic mixing matrix, the elements $Y_{\nu}^{\alpha \beta}$ of the neutrino Yukawa matrix must be drawn from a Gaussian distribution $\propto \exp \left[-\left|Y_{\nu}^{\alpha \beta}\right|^{2}\right]$, and the entries $M^{\alpha \beta}$ of the right-handed mass matrix must be drawn from a Gaussian distribution $\propto \exp \left[-\left|M^{\alpha \alpha}\right|^{2} / \mathcal{M}^{2}\right]$ for the diagonal elements and $\propto \exp \left[-2\left|M^{\alpha \beta}\right|^{2} / \mathcal{M}^{2}\right]$ for the off-diagonal elements [16]. Here, $\mathcal{M}$ is a dimensionful parameter that sets the overall mass scale of the right-handed neutrino. This way of generating random mass matrices will be used throughout this work. The statistical distribution of $\mathcal{M}$ does not follow from principles like basis independence and statistical independence of parameters, unlike the distribution of $\left|M^{\alpha \beta}\right| / \mathcal{M}$. Therefore, $\mathcal{M}$ must be considered an ad hoc choice, and consequently neutrino anarchy cannot make firm predictions for dimensionful quantities like the neutrino mass squared differences.

A suitable statistical test for quantifying the compatibility of the data with neutrino anarchy is the multidimensional Kolmogorov-Smirnov (KS) test [3, 5]. For a given oscillation parameter $X$, the test statistic

$$
D_{\mathrm{KS}}\left(x_{0}\right) \equiv \sup _{x^{\prime}}\left|\Theta\left(x^{\prime}-x_{0}\right)-F\left(x^{\prime}\right)\right|,
$$

measures the maximum distance between the theoretically predicted cumulative distribution function (CDF) $F\left(x^{\prime}\right)$ of $x$ and the experimentally determined approximation $\Theta\left(x^{\prime}-x_{0}\right)$ to the CDF. Since we have only one Universe to measure the neutrino oscillation parameters in, we have to contend ourselves with just one measurement $x_{0}$, therefore the experimental approximation to the CDF is simply a Heaviside step function. In our case, $X$ is one of the parameters appearing in the Haar measure (4), namely $\sin ^{2} \theta_{12}, \sin ^{2} \theta_{23}$ or $\cos ^{4} \theta_{13}$. Since the predicted distributions of these parameters are flat, we have $F\left(x^{\prime}\right)=x^{\prime}$. Thus, the test statistic is simply given by $D_{\mathrm{KS}}\left(x_{0}\right)=\frac{1}{2}+\left|\frac{1}{2}-x_{0}\right|$. The $p$-value

$$
p_{\mathrm{KS}}\left(x_{0}\right) \equiv 2\left[1-D_{\mathrm{KS}}\left(x_{0}\right)\right]=1-\left|1-2 x_{0}\right|
$$

gives the probability that, for a randomly drawn value $x^{\prime}$ of $X$, the test statistic $D_{\mathrm{KS}}\left(x^{\prime}\right)$ is larger than for the reference value $x_{0}$, i.e. that $D_{\mathrm{KS}}\left(x^{\prime}\right) \geq D_{\mathrm{KS}}\left(x_{0}\right)$. In other words, $p_{\mathrm{KS}}\left(x_{0}\right)$ measures the probability that the parameter takes a value more "extreme" (closer to the minimum and maximum values of 0 and 1) than the reference value $x_{0}$. Small $p_{\mathrm{KS}}$ means that $x_{0}$ is an unlikely outcome of the random draw, i.e. that the data does not support the anarchy hypothesis. For testing the compatibility of the anarchy hypothesis with not only the measurement of one parameter, but with measurements of all three mixing angles, we define the probability of the data as 


$$
\begin{array}{r}
P_{\mathrm{KS}} \equiv \int_{0}^{1} d \sin ^{2} \theta_{12} d \sin ^{2} \theta_{23} d \cos ^{4} \theta_{13} \Theta\left[p_{\mathrm{KS}}\left(\sin ^{2} \theta_{12}^{\mathrm{obs}}\right) p_{\mathrm{KS}}\left(\sin ^{2} \theta_{23}^{\mathrm{obs}}\right) p_{\mathrm{KS}}\left(\cos ^{4} \theta_{13}^{\mathrm{obs}}\right)\right. \\
\left.-p_{\mathrm{KS}}\left(\sin ^{2} \theta_{12}\right) p_{\mathrm{KS}}\left(\sin ^{2} \theta_{23}\right) p_{\mathrm{KS}}\left(\cos ^{4} \theta_{13}\right)\right] .
\end{array}
$$

Here, $\theta_{i j}^{\text {obs }}$ denotes the measured values of the oscillation parameters. Note that the statistical independence of the parameters appearing in the Haar measure implies that we can easily generalize this procedure to include additional parameters, for instance the Dirac CP phase $\delta_{\mathrm{CP}}$. Since the experimentally measured parameter values [17]

$$
\sin ^{2} \theta_{12}=0.304 \quad \sin ^{2} \theta_{23}=\left\{\begin{array}{l}
0.452(\mathrm{NH}) \\
0.579(\mathrm{IH})
\end{array} \quad \sin ^{2} \theta_{13}=0.0218,\right.
$$

depend on whether the mass ordering is assumed to be normal $(\mathrm{NH})$ or inverted $(\mathrm{IH})$, we define the mass hierarchy-weighted probability of the data under the anarchy hypothesis

$$
\tilde{P}_{\mathrm{KS}}=p_{\mathrm{NH}} P_{\mathrm{KS}, \mathrm{NH}}+p_{\mathrm{IH}} P_{\mathrm{KS}, \mathrm{IH}} .
$$

Here $p_{N H}\left(p_{I H}\right)$ denotes the probability of obtaining a normal (inverted) mass hierarchy in the anarchy scenario, and $P_{\mathrm{KS}, \mathrm{NH}}\left(P_{\mathrm{KS}, \mathrm{IH}}\right)$ is the Kolmogorov-Smirnov probability of the normal hierarchy (inverted hierarchy) best fit point, computed according to eq. (7). Note that $\tilde{P}_{\mathrm{KS}}$ is dominated by the first term since $p_{\mathrm{NH}} \sim 0.95$. When comparing neutrino anarchy predictions to data in the remainder of this paper, we will always use these weighted $\tilde{P}_{\mathrm{KS}}$ values.

Numerically, we find

$$
\tilde{P}_{\mathrm{KS}}=0.411 \text {. }
$$

This implies that the probability that a set of mixing angles randomly chosen according to the Haar measure gives worse $p$-values than the experimentally observed ones is $41.1 \%$. We can thus conclude that the data is well consistent with the anarchy hypothesis, in accord with ref. [5]. Figure 1 shows the value of $P_{\mathrm{KS}}$ as a function of $\sin ^{2} \theta_{12}^{\text {obs }}$ and $\sin ^{2} \theta_{23}^{\text {obs }}$ (left panel) and of $\sin ^{2} \theta_{23}^{\text {obs }}$ and $\sin ^{2} \theta_{13}^{\text {obs }}$ (right panel), with the third mixing angle fixed in both cases (see ref. [5] for similar plots).

In addition to the KS test, we have considered two additional statistical tests, namely the Anderson-Darling and the Cramér-von Mises tests. For our simple case where only one measurement is available and parameters have uniform distributions in the interval $[0,1]$, the test statistic for the Anderson-Darling test is

$$
D_{\mathrm{AD}}\left(x_{0}\right) \equiv-1-\log \left(1-x_{0}\right)-\log \left(x_{0}\right),
$$

and the one for the Cramér-von Mises test reads

$$
D_{\mathrm{CvM}}\left(x_{0}\right) \equiv \frac{1}{3}-x_{0}+x_{0}^{2} .
$$

It is, however, easy to see that the corresponding $p$-values $p_{\mathrm{AD}}\left(x_{0}\right)$ and $p_{\mathrm{CvM}}\left(x_{0}\right)$, i.e. the probabilities that a randomly drawn value $x^{\prime}$ leads to a larger value for the test statistic than the reference value $x_{0}$, are identical to the $p$-value for the KS test, eq. (6). Therefore, the Anderson-Darling and Cramer-von Mises tests do not provide any additional insights. In this context, let us also mention ref. [18, which discusses a Bayesian comparison of various anarchical models to models with flavor symmetries, finding consistence with both and a slight preference for the latter. Similar conclusions have been reached also in ref. [9]. 


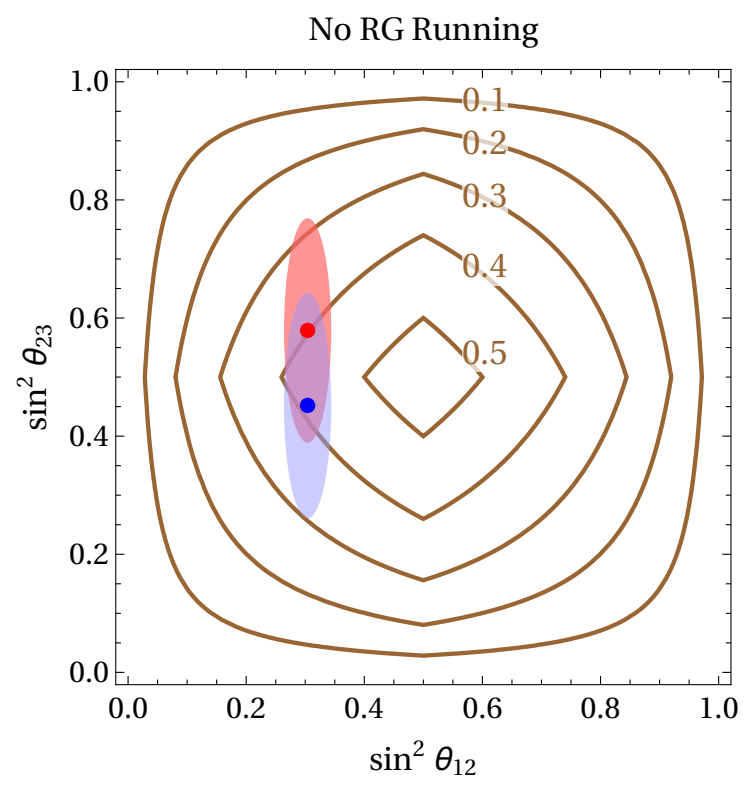

(a)

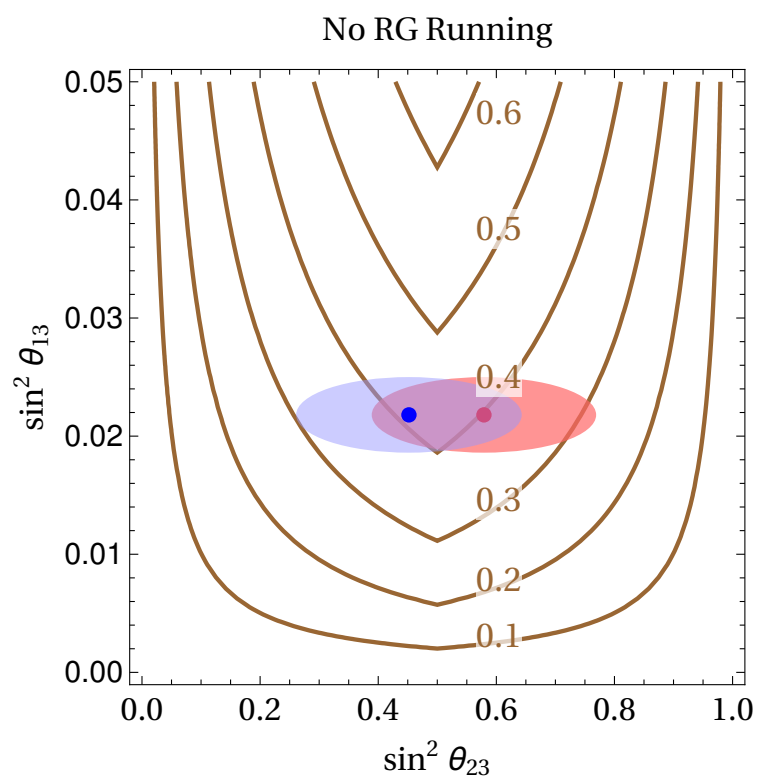

(b)

Figure 1. (a) Contours of equal probability $P_{\mathrm{KS}}$ (see eq. (7)) in the $\sin ^{2} \theta_{12}-\sin ^{2} \theta_{23}$ plane. The red (blue) ellipses show the favored region from the global fit in ref. [17 at the $3 \sigma$ confidence level for inverted (normal) mass ordering. The colored dots indicate the corresponding best fit points. We have fixed the third mixing angle at its best fit value, $\sin ^{2} \theta_{13}=0.0218$. (b) Contours of equal probability $P_{\mathrm{KS}}$ in the $\sin ^{2} \theta_{23}-\sin ^{2} \theta_{13}$ plane, compared to the experimentally allowed regions for both mass orderings (same coloring as in (a)). We have fixed the solar mixing angle at its best fit value, $\sin ^{2} \theta_{12}=0.304$.

Even though the predictions of the neutrino anarchy scenario depend on the ad hoc choice of the right-handed mass scale $\mathcal{M}$, it is possible to draw non-trivial conclusions on the relations between different neutrino mass eigenvalues. A particularly interesting quantity to consider in this context is the effective Majorana mass measured in neutrinoless double beta decay,

$$
\left|m_{e e}\right| \equiv\left|\sum_{j} U_{e j}^{2} m_{j}\right|
$$

where $U_{e j}$ are the elements in the first row of the leptonic mixing matrix and $m_{j}$ are the light mass eigenvalues. (In this paper, we always assume neutrinos to have Majorana masses, so that neutrinoless double beta decay is in principle possible.) We will therefore also discuss how neutrinoless double beta decay experiments can probe the predictions of anarchy in the future, and how these predictions are affected by renormalization group running.

\section{RENORMALIZATION GROUP EVOLUTION AND ANARCHY}

Like all Lagrangian parameters in a quantum field theory, the neutrino masses and mixing parameters are subject to renormalization group running. It follows that, when comparing the predicted probability distribution of a parameter to its measured value, we have to be careful to evaluate both at the same renormalization scale. In particular, neutrino oscillation measurements are typically carried out at energy scales of order $\mathrm{MeV}-\mathrm{GeV}$, while the flavor structure of a typical seesaw model depends on scales $M_{\mathrm{GUT}} \sim 10^{16} \mathrm{GeV}$. Therefore, we will in the following repeat the analysis of neutrino anarchy, but now carefully taking into account renormalization group effects. 


\section{III.1. Type-I Seesaw Model}

The first example we will study is the type-I seesaw scenario, which extends the Standard Model by three heavy gauge singlet neutrinos with Majorana masses of $\mathcal{O}\left(10^{14} \mathrm{GeV}\right)$, which couple to the active SM neutrinos via a Yukawa coupling [19, 20]. The Lagrangian is given by eq. (1).

The renormalization group equations for the type-I seesaw model have been studied in great detail in refs. [21 25. To investigate the effect of RG evolution on neutrino anarchy in this model, we have randomly generated $10^{5}$ sets of parameters, defined by the Yukawa matrices $Y_{\nu}$ and the right-handed neutrino mass matrices $M$. We assume these randomly generated matrices to enter the Lagrangian at the high scale $M_{\mathrm{GUT}}=10^{16} \mathrm{GeV}$. As explained in ref. [16], to ensure basis invariance of the probability distributions and statistical independence of different mass matrix elements, the elements of $Y_{\nu}^{\alpha \beta}$ must follow a Gaussian distribution $\propto \exp \left[-\left|Y_{\nu}^{\alpha \beta}\right|^{2} / \mathcal{Y}\right]$. The diagonal elements $M^{\alpha \alpha}$ of the right-handed Majorana mass matrix follow a similar distribution $\propto \exp \left[-\left|M^{\alpha \alpha}\right|^{2} / \mathcal{M}\right]$, while the distribution of the off-diagonal elements $M^{\alpha \beta}$ of $M$ is $\propto \exp \left[-2\left|M^{\alpha \beta}\right|^{2} / \mathcal{M}\right]$. Here $\mathcal{Y}$ is a dimensionless parameter which we choose to be one, while $\mathcal{M}$ is dimensionful, and we choose $\mathcal{M}=10^{14} \mathrm{GeV}$ to reproduce the correct order of magnitude for the light neutrino masses. Below we will discuss the impact of different choices for $\mathcal{M}$.

For the parameters of the standard model at the high scale we have chosen a set of values offered by the REAP package [21]:

$$
\begin{aligned}
& Y_{u}\left(M_{\mathrm{GUT}}\right)=\left(\begin{array}{lll}
5.4039 \cdot 10^{-6} & & \\
& 1.5637 \cdot 10^{-3} & \\
& & 0.4829
\end{array}\right), \\
& Y_{d}\left(M_{\mathrm{GUT}}\right)=10^{-5} \times\left(\begin{array}{ccc}
2.1176 & 4.5697+0.0112 i & 2.1367+0.2581 i \\
4.5697-0.0112 i & 22.3698 & 25.7097-0.0019 i \\
2.1367-0.2571 i & 25.7097+0.0019 i & 614.0010
\end{array}\right) \text {, } \\
& Y_{e}\left(M_{\mathrm{GUT}}\right)=\left(\begin{array}{lll}
2.8370 \cdot 10^{-6} & & \\
& 0.5988 \cdot 10^{-3} & \\
& & 10.1789 \cdot 10^{-3}
\end{array}\right) \text {. }
\end{aligned}
$$

Of course the low-scale values of the quark and charged lepton Yukawa matrices depend on the additional field content beyond the SM and on the values of the new parameters introduced. Therefore, we typically cannot reproduce the SM Yukawa couplings exactly, with deviations being of order $10 \%$. Nevertheless, since our interest here is in the running of the neutrino masses and mixing parameters, these small deviations are negligible for our purposes.

We evolve each parameter point down to a lower scale, which we take to be $M_{Z}=91.19 \mathrm{GeV}$. In principle, we should evolve the mixing parameters down to an even lower scale, integrating out SM particles along the way. However, as we will see, the dominant contribution to the running of the mixing parameters arises at scales $\gg M_{Z}$ and thus it is justified to stop the RG evolution at $M_{Z}$. The renormalization group equations for the type-I seesaw scenario have been derived in [21], and we follow the exact same procedure. Both at $M_{\mathrm{GUT}}$ and at $M_{Z}$, we extract the values of the physical mixing parameters by diagonalizing the mass matrices and then following appendix A of ref. [14]. In diagonalizing $m_{\nu}$, we have to specify a convention for the ordering of the mass eigenvalues. We always choose them to be in ascending order (case A3 in ref. [15]). Note that for the running of the neutrino masses, decoupling effects cannot be neglected and even the top-quark decoupling may become important [26]. However, since we are mostly interested in the running of the mixing angles and CP phases here, it is justified to neglect decoupling effects. This is especially true for the top and the Higgs because the running of the mixing parameters is negligible at their mass scale (see e.g. fig. 4 below). 


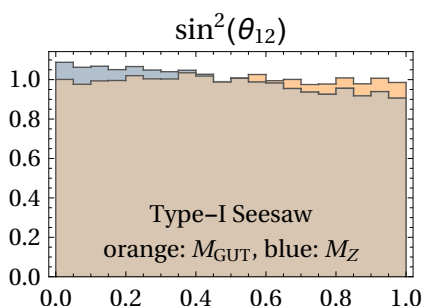

(a) Distribution of $\sin ^{2} \theta_{12}$

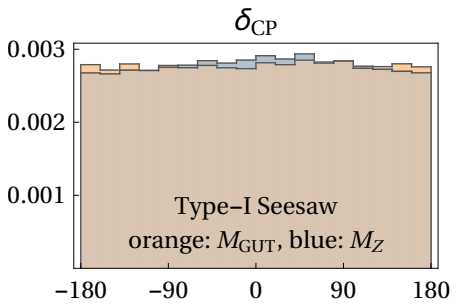

(d) Distribution of $\left.\delta_{\mathrm{CP}}{ }^{\circ}\right]$

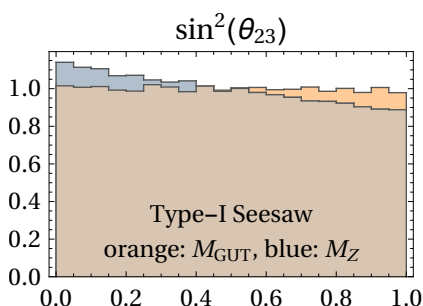

(b) Distribution of $\sin ^{2} \theta_{23}$

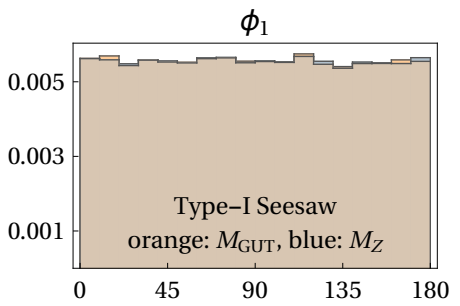

(e) Distribution of $\phi_{1}\left[^{\circ}\right]$

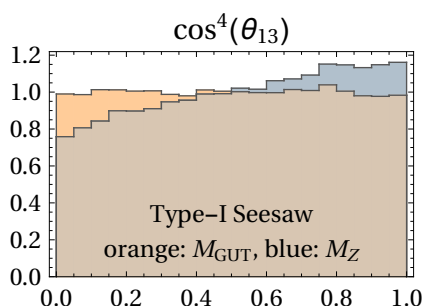

(c) Distribution of $\cos ^{4} \theta_{13}$

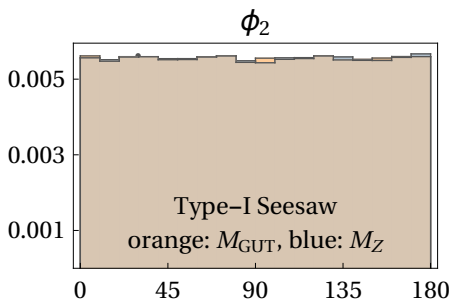

(f) Distribution of $\phi_{2}\left[^{\circ}\right]$

Figure 2. Distributions of the three mixing angles, the Dirac CP phase $\delta_{\mathrm{CP}}$ and the two Majorana CP phases $\phi_{1}$ and $\phi_{2}$ before and after renormalization group running in the type-I seesaw model. Orange regions correspond to parameters at $M_{\mathrm{GUT}}$, blue regions to parameters at $M_{Z}$. Note that the physical range for $\delta_{\mathrm{CP}}$ is $\left[-180^{\circ}, 180^{\circ}\right)$, while the physical ranges for $\phi_{1}$ and $\phi_{2}$ are only $\left[0,180^{\circ}\right)$ [15].

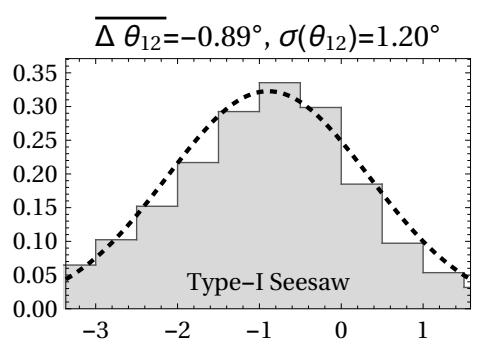

(a) Distribution of $\Delta \theta_{12}\left[^{\circ}\right]$

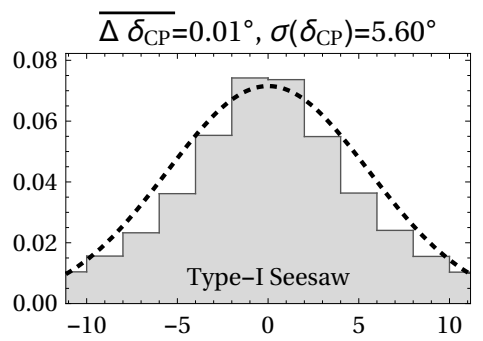

(d) Distribution of $\Delta \delta_{\mathrm{CP}}\left[^{\circ}\right]$

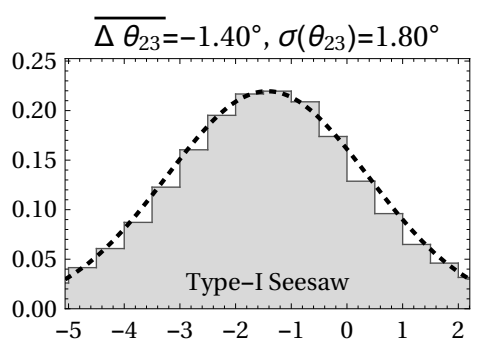

(b) Distribution of $\Delta \theta_{23}\left[^{\circ}\right]$

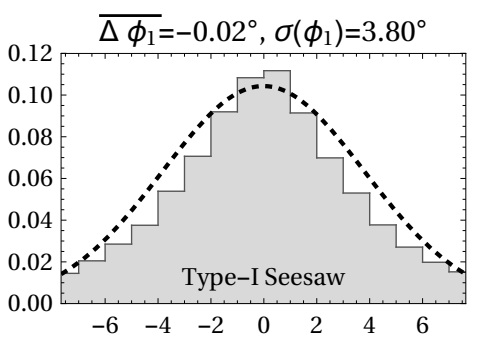

(e) Distribution of $\Delta \phi_{1}\left[{ }^{\circ}\right]$

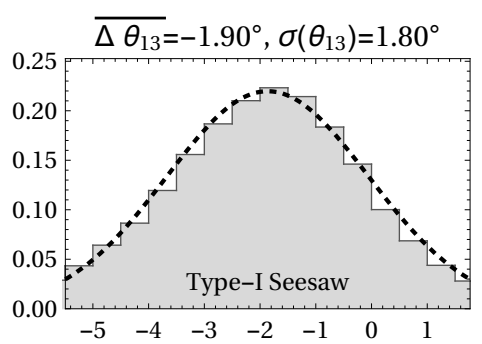

(c) Distribution of $\Delta \theta_{13}\left[{ }^{\circ}\right]$

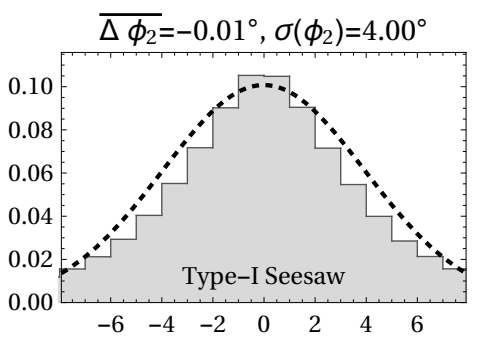

(f) Distribution of $\Delta \phi_{2}\left[{ }^{\circ}\right]$

Figure 3. Distributions of the RG-induced shifts in the mixing parameters, $\Delta x \equiv x\left(M_{Z}\right)-x\left(M_{\mathrm{GUT}}\right)$ in the type-I seesaw model. Also shown are the central values $\overline{\Delta x}$ and the widths $\sigma(x)$ of Gaussian fits to the distributions.

We show our results in fig. 2. In each panel, we compare the distribution of one of the mixing parameters at $M_{\mathrm{GUT}}$ (orange histograms) to the distribution at $M_{Z}$ after RG running (blue histograms). By construction, the distributions at $M_{\mathrm{GUT}}$ are flat, while at $M_{Z}$, we observe a small bias towards smaller mixing angles. We show in fig. 3 the distributions of the RG-induced shifts, $\Delta x=x\left(M_{Z}\right)-x\left(M_{\mathrm{GUT}}\right)$, where $x \in\left\{\theta_{12}, \theta_{13}, \theta_{23}, \delta_{\mathrm{CP}}, \phi_{1}, \phi_{2}\right\}$. We have also fitted each histogram 


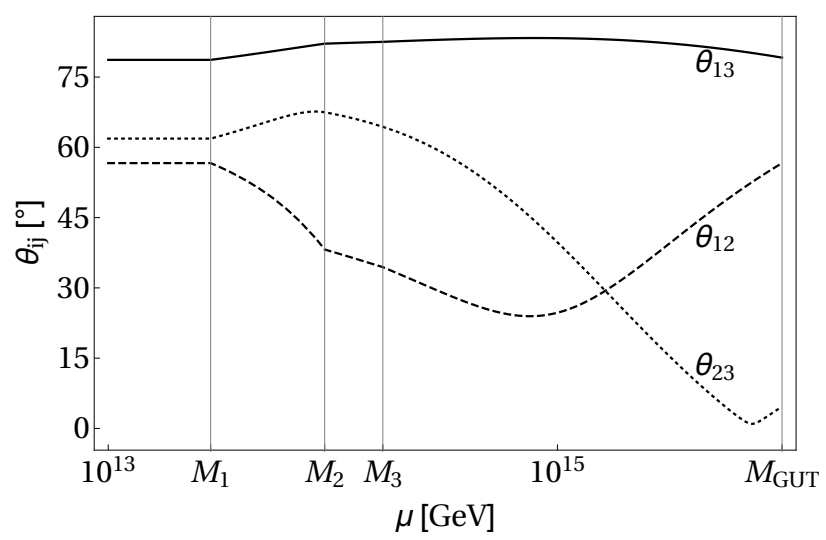

Figure 4. A parameter point of the type-I seesaw model with large running effects in the neutrino mixing angles. The vertical dotted lines show the decoupling thresholds of the heavy singlet neutrinos, $M_{1}=$ $2.9 \cdot 10^{13} \mathrm{GeV}, M_{2}=9.2 \cdot 10^{13} \mathrm{GeV}$ and $M_{3}=1.67 \cdot 10^{14} \mathrm{GeV}$. Note how RG running ceases after the last heavy neutrino is integrated out. The masses of the active neutrinos at the low scale are $m_{1}=0.02 \mathrm{eV}$, $m_{2}=0.32 \mathrm{eV}$ and $m_{3}=0.36 \mathrm{eV}$.

in fig. 3 to a Gaussian distribution

$$
f(x)=\frac{1}{\sqrt{2 \pi} \sigma(x)} \exp \left[-\frac{(x-\bar{x})^{2}}{2[\sigma(x)]^{2}}\right],
$$

and we give the fitted values of $\bar{x}$ and $\sigma(x)$ in the plots.

We find a slight preference for the mixing angles to decrease by $1-2^{\circ}$ after evolving them from $M_{\mathrm{GUT}}$ to $M_{Z}$ : the distributions of $\Delta \theta_{i j}$ all peak at small negative values. The distribution of the Dirac CP phase $\delta_{\mathrm{CP}}$ in fig. 2 (d) shows a slight peak at zero after running, while the distributions of the Majorana phases $\phi_{1}$ and $\phi_{2}$ in fig. 2 (e), (f) are flat both at $M_{\mathrm{GUT}}$ and at $M_{Z}$. These are of course only statements about the average behavior of a large set of random parameter pointsindividual points can lead to much larger running effects. In particular, for scenarios where at least two neutrino mass eigenvalues are very close to each other, the running of the mixing angles is known to be potentially large [21].

Even for parameter points that exhibit significant RG running, we observe that most of the running happens at scales above the masses of the heavy singlet neutrinos. Figure 4 shows an example where the mixing angles exhibit large RG running. We observe that the running gradually diminishes as the heavy singlet neutrinos are integrated out and completely ceases below the mass of the lightest of them. This observation allows us to draw conclusions on the impact of the right-handed neutrino mass scale $\mathcal{M}$ on the RG evolution: For $\mathcal{M}$ larger (smaller) than our choice $\mathcal{M}=10^{14} \mathrm{GeV}$, the singlet neutrinos are typically heavier (lighter), so the running ceases sooner (later), and the overall shift in the parameters will be smaller (larger). We explicitly checked this behavior numerically.

The fact that the mixing angles run preferentially downwards can be understood in the following way. First, recall that the leptonic mixing matrix depends on the matrix $V_{\nu}$ that diagonalizes $m_{\nu}$ and on the matrix $V_{e}$ that diagonalizes $Y_{e}^{\dagger} Y_{e}$ according to $V_{e} Y_{e}^{\dagger} Y_{e} V_{e}^{\dagger}=D$, where $D$ is diagonal. The flavor non-trivial terms in the $\beta$ functions for the mass and Yukawa matrices (see appendix A) are dominated by the $\mathcal{O}(1)$ terms containing $Y_{\nu}$. The subdominant terms involving $Y_{e}$ are negligible. Since $Y_{\nu}$ is randomly chosen, it cannot lead to a preference for one sign over the other in the $\beta$ function. Thus, the only possible source of such a preference can come from the initial conditions 
for $Y_{e}$. The $\beta$ function for $Y_{e}$ reads

$$
16 \pi^{2} \beta_{Y_{e}}=-\frac{3}{2} Y_{e}\left(Y_{\nu}^{\dagger} Y_{\nu}\right)+\ldots
$$

where the dots indicate the subdominant and flavor-diagonal terms. The initial condition $Y_{e}\left(M_{\mathrm{GUT}}\right)$ for $Y_{e}$ is a diagonal matrix (see eq. (14)), with its strongly hierarchical eigenvalues sorted in ascending order. The structure of eq. (16) shows that after RG running, the elements in the $k$-th row of $Y_{e}$ will change by an amount proportional to the $(k k)$ elements of $Y_{e}\left(M_{\mathrm{GUT}}\right)$. To proceed, it is easiest to consider a two flavor system for illustrative purposes. In this case, using the hierarchy of the elements of $Y_{e}$ after running, one can show that the mixing angle parameterizing $V_{e}$ is positive. Since $U_{\mathrm{PMNS}}=V_{e}^{\dagger} V_{\nu}$, and since the mixing angle describing $V_{\nu}$ is random in $[-\pi, \pi]$, the mixing angle describing $U_{\mathrm{PMNS}}$ is then preferentially negative. In models where the dominant term in (16) comes with a different sign, the preferred direction of the running would be reversed. One example of such model is a type-II seesaw model [27].

To study how RG running affects the compatibility of neutrino anarchy with the observed mixing parameters, we use again the Kolmogorov-Smirnov test. Note that the multidimensional KS test described in section II is only meaningful if the parameters in the integral eq. (7) are statistically independent and follow a flat distribution. We have checked that, even after RG running, the parameters $\sin ^{2} \theta_{12}, \sin ^{2} \theta_{23}$ and $\cos ^{4} \theta_{13}$ are still nearly uncorrelated. For instance, the modulus of Pearson's correlation coefficient, $|\langle x \cdot y\rangle /(\langle x\rangle\langle y\rangle)|$, is $\lesssim 0.02$ for all combinations of $x, y=\sin ^{2} \theta_{12}, \sin ^{2} \theta_{23}, \cos ^{4} \theta_{13}$. To obtain flat distributions, we define new parameters $\sin ^{2} \theta_{12}^{\prime}$, $\sin ^{2} \theta_{23}^{\prime}$ and $\cos ^{4} \theta_{13}^{\prime}$ in the following way: we fit each of the distributions in fig. 2 (a), (b), (c) by a cubic polynomial $f(x)$, where $x=\sin ^{2} \theta_{12}, \sin ^{2} \theta_{23}, \cos ^{4} \theta_{13}$. We normalize $f(x)$ such that its integral over the physical range of $x$ is 1 . We then define $x^{\prime}(x) \equiv \int_{0}^{x} d \tilde{x} f(\tilde{x})$. Replacing the unprimed variables $x$ in eq. (7) by the primed ones $x^{\prime}(x)$, the KS test can now be applied in a meaningful way.

A further subtlety arises because the RG evolution is different for parameter points with a normal mass ordering and parameter points with an inverted mass ordering. We therefore separate our randomly generated points according to the predicted mass ordering and compute $P_{\mathrm{KS}, \mathrm{NH}}$ $\left(P_{\mathrm{KS}, \mathrm{IH}}\right)$ in eq. $(9)$ using only points with normal (inverted) ordering. We find that the probability $\tilde{P}_{\mathrm{KS}}$ of the observed parameter values, given the anarchy hypothesis, increases by $\sim 7 \%$ from $41.1 \%$ to $47.7 \%$ due to RG effects. Note that, as before, $\tilde{P}_{\mathrm{KS}}$ is entirely dominated by parameter points with a normal mass ordering because the probability of obtaining an inverted ordering from anarchy is only at the $5 \%$ level. We show in fig. 5 the contours of equal Kolmogorov-Smirnov probability $P_{\mathrm{KS}, \mathrm{NH}}$ for parameter points with normal mass ordering before and after running.

Finally, we also comment on the running of the neutrino mass parameters, which we find to be substantial. Parameterizing their approximate evolution as $d m / d \log \mu=-\gamma m$, we find for the anomalous dimension of the three light mass eigenvalues an average value of $\gamma \simeq-0.027$. This implies that the light neutrino masses run down by about $60 \%$ between $M_{\mathrm{GUT}}$ and $M_{Z}$.

We investigate in fig. 6 what neutrino anarchy with and without RG running predicts for the outcome of future neutrinoless double beta $(0 \nu 2 \beta)$ decay experiments. Of course, since the scale choice $\mathcal{M}$ for the right-handed neutrinos is an input parameter even in the anarchy scenario, the absolute scale of $m_{\text {lightest }}$ and $m_{e e}$ can be chosen at will. In fact, the left and right panels in fig. 6 represent two different choices for $\mathcal{M}$. Nonetheless, we can draw several important conclusions from fig. 6. First, we see again that anarchy favors the normal mass ordering: there are much fewer points in panels (c) and (d) than in panels (a) and (b). In other words, if a future $0 \nu 2 \beta$ search finds a result compatible only with an inverted mass ordering, this would disfavor anarchy. Second, we see that scenarios with relatively large $m_{e e}$ but very small $m_{\text {lightest }}$, such as the leftmost part of the inverted hierarchy band, are disfavored, as are scenarios with very small $m_{e e}$ but sizeable $m_{\text {lightest }}$, 


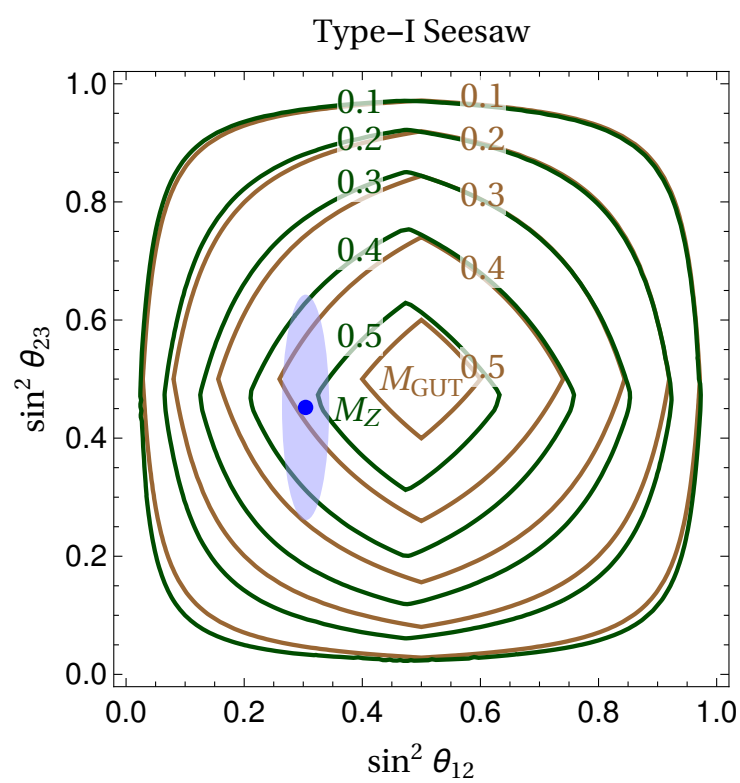

(a)

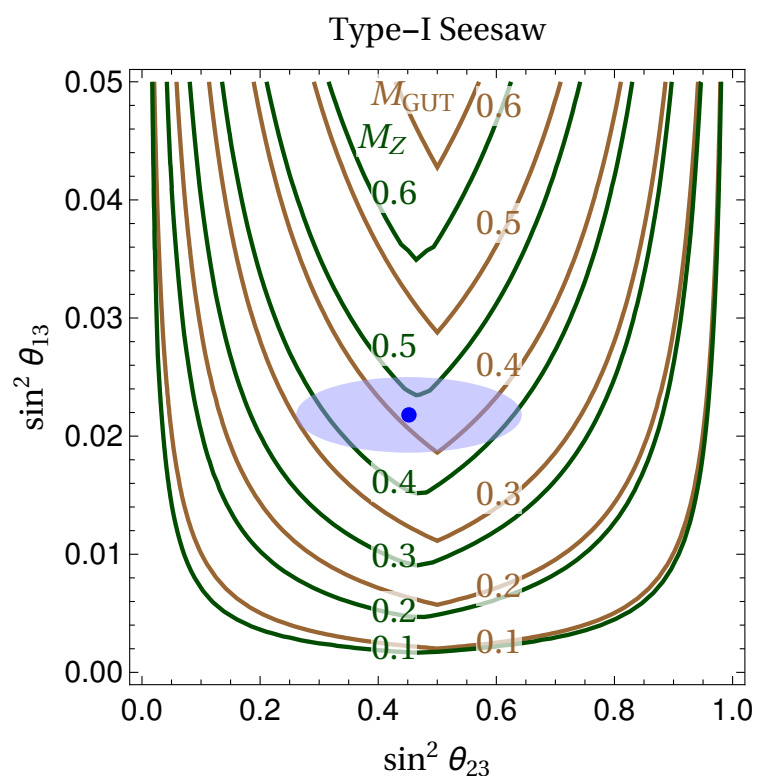

(b)

Figure 5. (a) Contours of equal probability $P_{\mathrm{KS}, \mathrm{NH}}$ (see eqs. (7) and (9) in the $\sin ^{2} \theta_{12}-\sin ^{2} \theta_{23}$ plane with RG effects included (dark green) and without RG effects (brown, see fig. 1 (a)) for the type-I seesaw model. We consider only parameter points with a normal mass ordering (see text for details). The blue ellipse indicates the normal ordering favored region from the global fit in ref. [17] at the $3 \sigma$ confidence level, and the blue dot is the corresponding best fit point. We have fixed the third mixing angle at its best fit value, $\sin ^{2} \theta_{13}=0.0218$. With RG effects included, the likelihood of the data with respect to anarchy increases from $41.1 \%$ to $47.7 \%$. (b) Contours of equal probability $P_{\mathrm{KS}, \mathrm{NH}}$ in the $\sin ^{2} \theta_{23}-\sin ^{2} \theta_{13}$ plane with and without RG effects included (same coloring as in (a)). We have fixed the solar mixing angle at its best fit value, $\sin ^{2} \theta_{12}=0.304$.

in particular the funnel region in the normal hierarchy case. Finally, also a quasi-degenerate mass spectrum is disfavored by the anarchy hypothesis. If a combination of $0 \nu 2 \beta$ experiments, cosmology and direct neutrino mass measurements should point to one of these disfavored regions in the future, the anarchy hypothesis would come under pressure.

\section{III.2. Inverse Seesaw Model}

As a second example, we consider the inverse seesaw scenario [32, 33], in which the SM Lagrangian is extended by

$$
\mathcal{L}_{\text {inverse seesaw }} \supset-\left(Y_{\nu}^{\dagger}\right)^{\alpha \beta} \bar{L}^{\alpha} \tilde{H} N_{R}^{\beta}-M_{D}^{\alpha \beta} \bar{S}^{\alpha}\left(N_{R}^{\beta}\right)^{c}-\frac{1}{2} m_{s}^{\alpha \beta} \bar{S}^{\alpha}\left(S^{\beta}\right)^{c}+\text { h.c. },
$$

where two types of new gauge singlet Weyl fermions are introduced: $n_{N}$ generations of $N_{R}$ fields, which participate directly in the Yukawa couplings, and $n_{S}$ generations of $S$ fields. Possible completions of the model that explain this structure for the Lagrangian are given in [34 37]. After electroweak symmetry breaking, the neutrino mass term becomes:

$$
\mathcal{L}_{m, \text { inverse seesaw }} \supset-\left(\overline{\nu_{L}^{c}}, \bar{N}_{R}, \bar{S}\right)\left(\begin{array}{ccc}
0 & Y_{\nu}^{T} v / \sqrt{2} & 0 \\
Y_{\nu} v / \sqrt{2} & 0 & M_{D}^{T} \\
0 & M_{D} & m_{s}
\end{array}\right)\left(\begin{array}{c}
\nu_{L} \\
N_{R}^{c} \\
S^{c}
\end{array}\right) .
$$




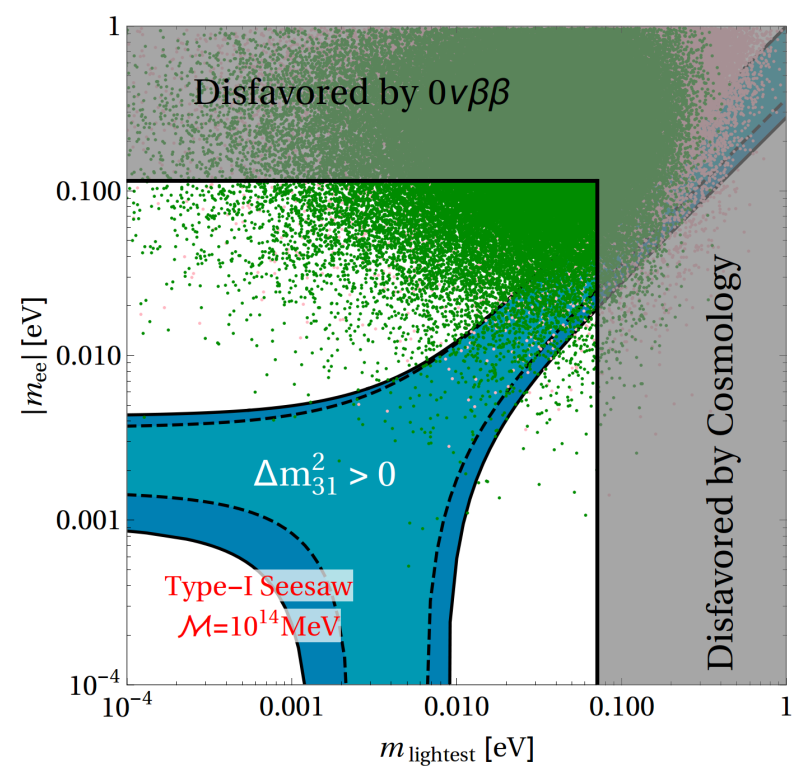

(a)

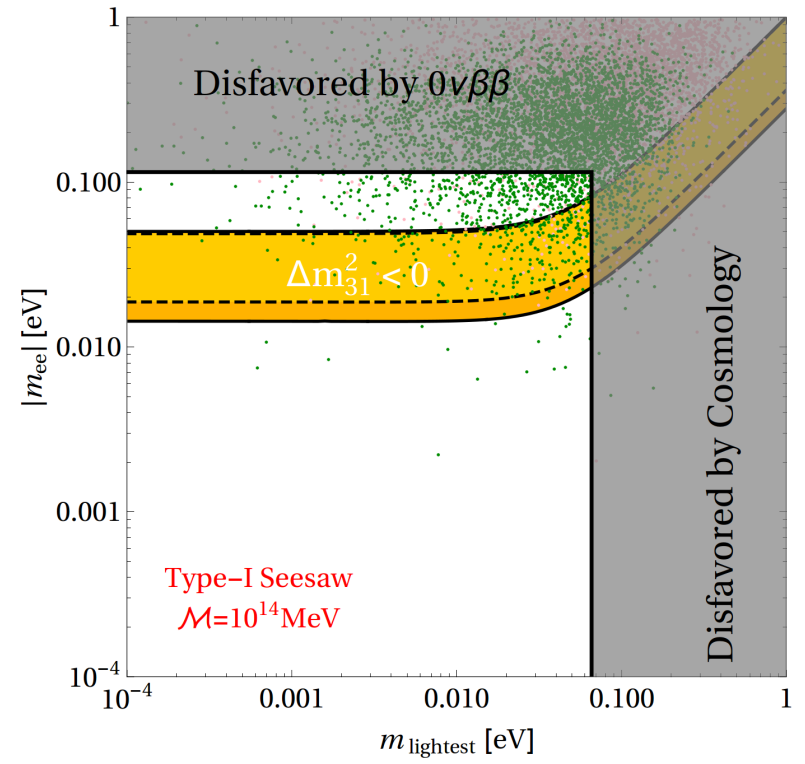

(c)

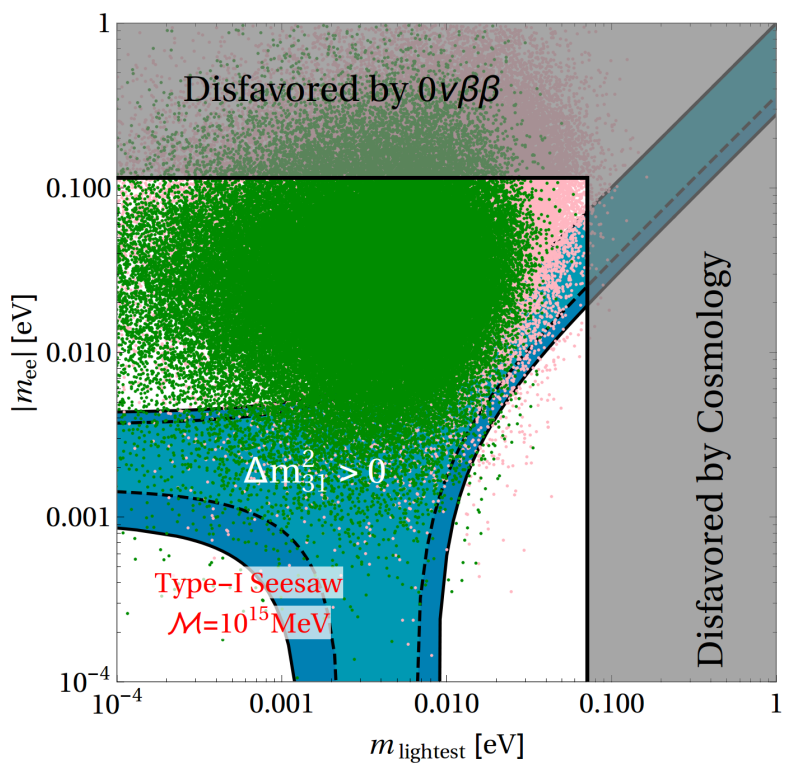

(b)

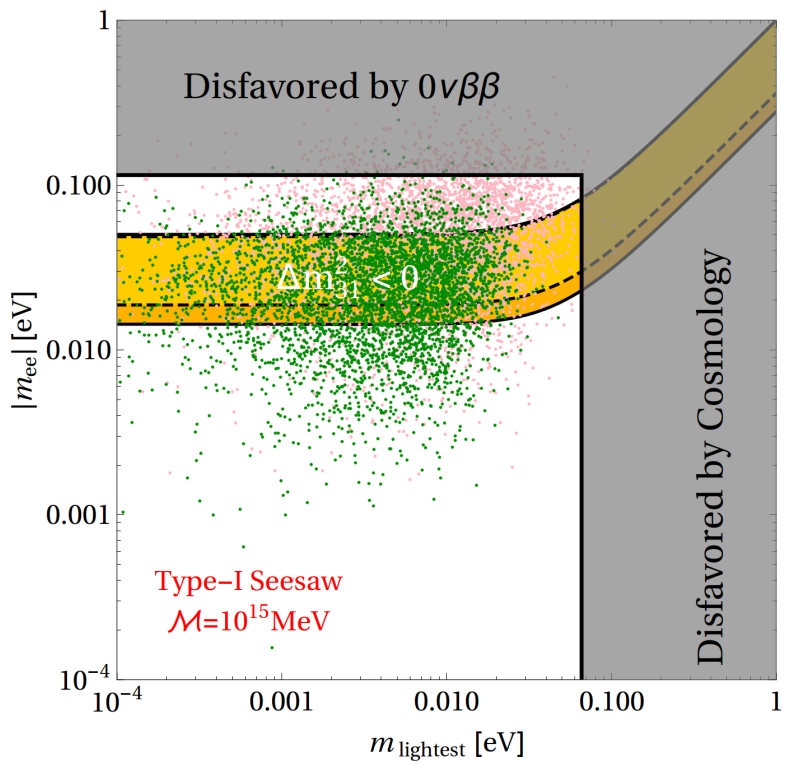

(d)

Figure 6. Colored dots: predictions for the effective mass $\left|m_{e e}\right|$ measured in neutrinoless double beta decay from the neutrino anarchy scenario in the type-I seesaw model. The mass scale of the right-handed neutrinos was set to $\mathcal{M}=10^{14} \mathrm{GeV}$ in the left panels, and to $\mathcal{M}=10^{15} \mathrm{GeV}$ in the right panels. Among the randomly generated parameter points, we distinguish those resulting in a normal neutrino mass ordering (upper panels) and those resulting in an inverted ordering (lower panels). Pink points show the distributions at $M_{\mathrm{GUT}}$, while green points show the shifted distributions after renormalization group evolution to $M_{Z}$. The colored bands show the allowed regions in the $m_{\text {lightest }}-\left|m_{e e}\right|$ plane from the global experimental data on the neutrino mass squared differences and mixing angles. Dashed lines correspond to varying only the unknown phases in $U_{\mathrm{PMNS}}$, while solid lines also include the uncertainties in the mixing angles. Note that, to be true to the spirit of anarchy, we do not impose any constraints on our randomly generated points, therefore a sizeable portion of them fall into regions already excluded by experiment. Gray regions are exclusion limits from cosmology [28] and from experimental searches for neutrinoless double beta decay [29]31. 


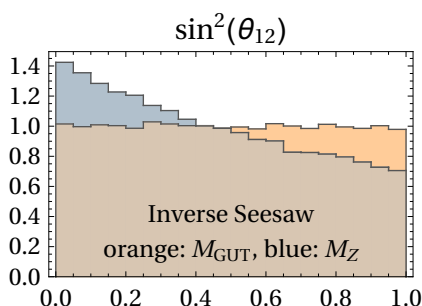

(a) Distribution of $\sin ^{2} \theta_{12}$

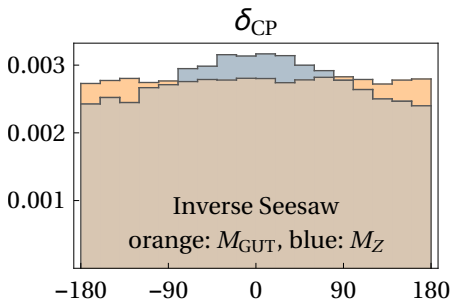

(d) Distribution of $\left.\delta_{\mathrm{CP}}{ }^{\circ}\right]$

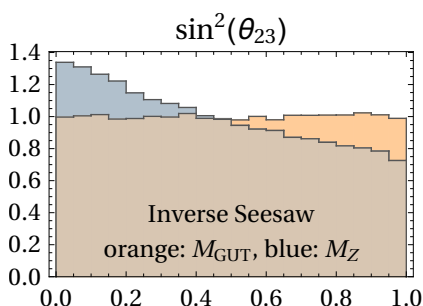

(b) Distribution of $\sin ^{2} \theta_{23}$

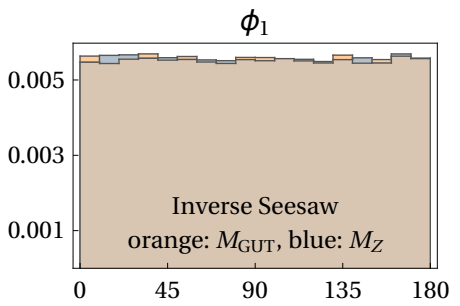

(e) Distribution of $\phi_{1}\left[^{\circ}\right]$

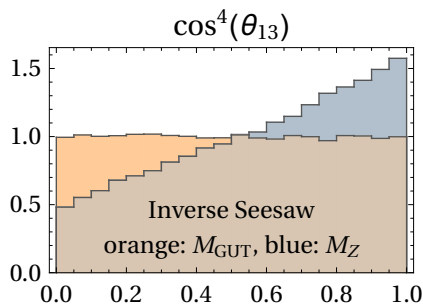

(c) Distribution of $\cos ^{4} \theta_{13}$

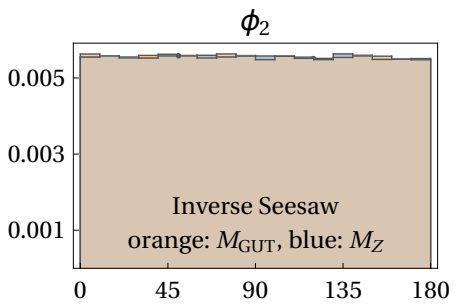

(f) Distribution of $\phi_{2}\left[^{\circ}\right]$

Figure 7. Distributions of the mixing angles and physical CP phases before and after renormalization group running in the inverse seesaw model. The orange regions correspond to the parameters at $M_{\mathrm{GUT}}$, the blue regions to the parameters at $M_{Z}$. As for the type-I seesaw model, the distributions of the Majorana CP phases are unaffected by RG evolution. For the mixing angles and the Dirac CP phase, the running is much stronger than in the type-I seesaw case.

For the effective Majorana mass matrix of the light active neutrinos we then obtain:

$$
m_{\nu} \simeq \frac{v^{2}}{2} Y_{\nu}^{T} M_{D}^{-1} m_{s} M_{D}^{-1} Y_{\nu}
$$

The correct neutrino mass eigenvalues $\lesssim \mathcal{O}(\mathrm{eV})$ are obtained for $M_{D} \sim \mathcal{O}(\mathrm{TeV}), m_{s} \sim \mathcal{O}(\mathrm{keV})$. The model has the additional virtue that, if the number of generations of $S$ is larger than the number of generations of $N_{R}, \mathcal{O}(\mathrm{keV})$ sterile mass eigenstates are obtained [38], which could act as the dark matter in the Universe. (If also $N_{R}$ has a Majorana mass term, the condition $n_{S}>n_{N}$ can be avoided, see e.g. ref. [39].) The RG equations for the inverse seesaw model are given in appendix A. They agree with the ones given in [40.

Carrying out an RG analysis on a random sample of $10^{5}$ mass matrices of the form of eq. 18, defined at $M_{\mathrm{GUT}}$ and evolved down to $M_{Z}$, we obtain the results shown in figs. 7 and 8 . The shifts in the mixing angles show the same characteristics as in the type-I seesaw model discussed in the previous section. In particular, mixing angles are preferentially shifted to smaller values, and $\delta_{\mathrm{CP}}$ is preferentially close to zero. The average magnitude of the shifts is, however, larger than in the type-I seesaw case: of order $4^{\circ}$ for $\theta_{12}$ and $\theta_{23}$, and of order $5.5^{\circ}$ for $\theta_{13}$. Also the widths of the distributions in fig. 8 are several times larger than they were for the type-I seesaw model (see fig. 3). The average anomalous dimension describing the running of the light neutrino masses is -0.037 in the inverse seesaw scenario, corresponding to a $70 \%$ decrease of the mass eigenvalues between $M_{\mathrm{GUT}}$ and $M_{Z}$.

To see how RG effects influence the consistency of the neutrino oscillation data with the predictions of anarchy, we compare in fig. 9 the equiprobability contours with and without RG running in the inverse seesaw model. To produce this plot, we follow the same procedure as for the type-I seesaw model (see section III.1). We exploit again the fact that the mixing parameters are still nearly uncorrelated after RG evolution, even though the absolute values of the correlation coefficients are now somewhat larger, $\sim 0.07$ now. We find that RG effects change the compatibility of 


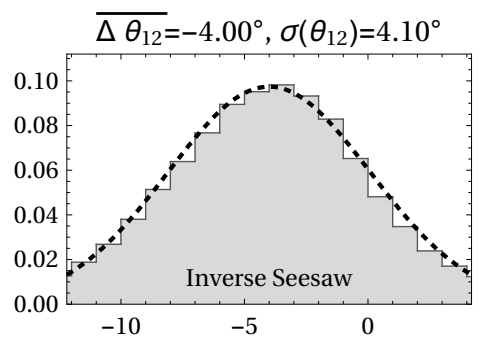

(a) Distribution of $\Delta \theta_{12}\left[^{\circ}\right]$

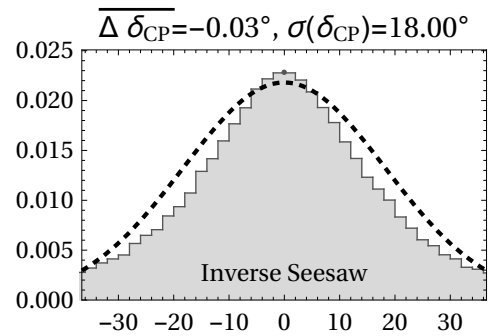

(d) Distribution of $\Delta \delta_{\mathrm{CP}}\left[^{\circ}\right]$

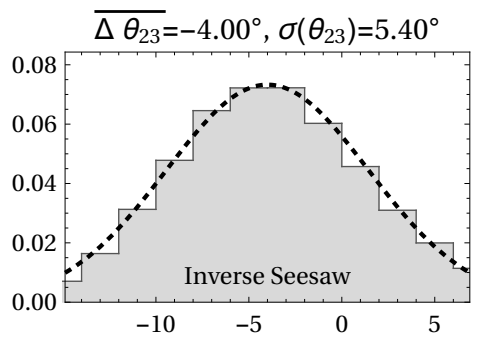

(b) Distribution of $\Delta \theta_{23}\left[^{\circ}\right]$

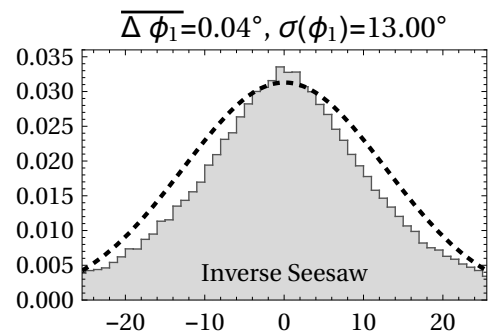

(e) Distribution of $\Delta \phi_{1}\left[^{\circ}\right]$

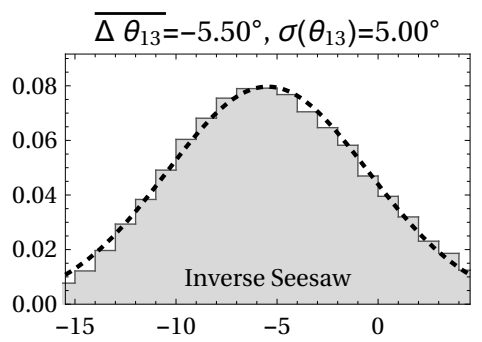

(c) Distribution of $\Delta \theta_{13}\left[^{\circ}\right]$

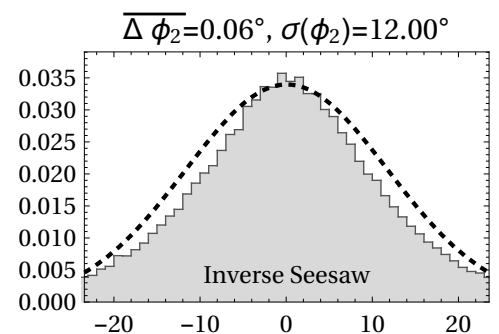

(f) Distribution of $\Delta \phi_{2}\left[^{\circ}\right]$

Figure 8. Shift in the mixing angles and the CP phases during RG evolution form $M_{\mathrm{GUT}}$ to $M_{Z}$ in the inverse seesaw model, obtained from $10^{5}$ randomly generated mass matrices. We use the notation $\Delta x \equiv$ $x\left(M_{Z}\right)-x\left(M_{\mathrm{GUT}}\right)$, where $x$ stands for any of the mixing angles or complex phases. Also shown are Gaussian fits with central values $\overline{\Delta x}$ and widths $\sigma(\Delta x)$.

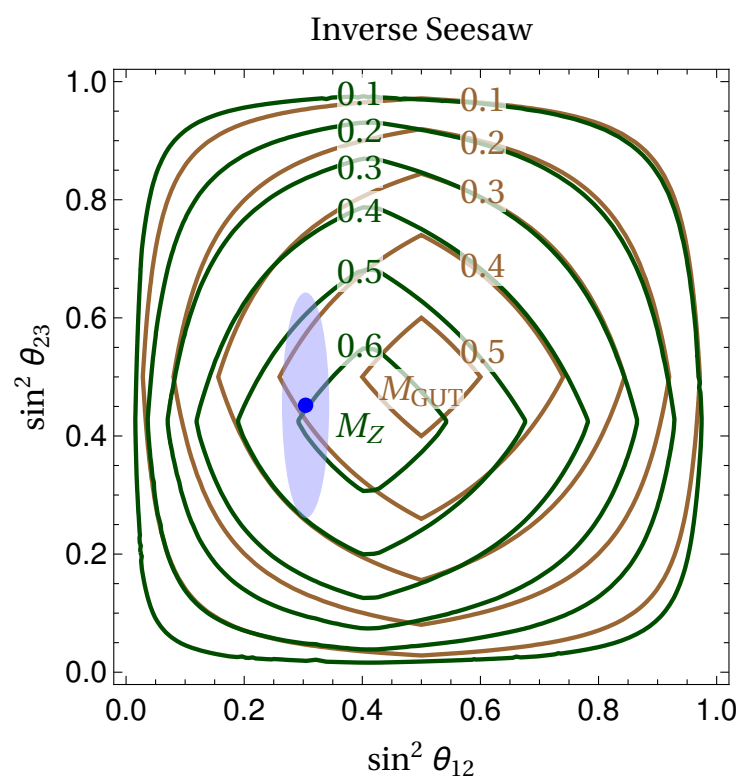

(a)

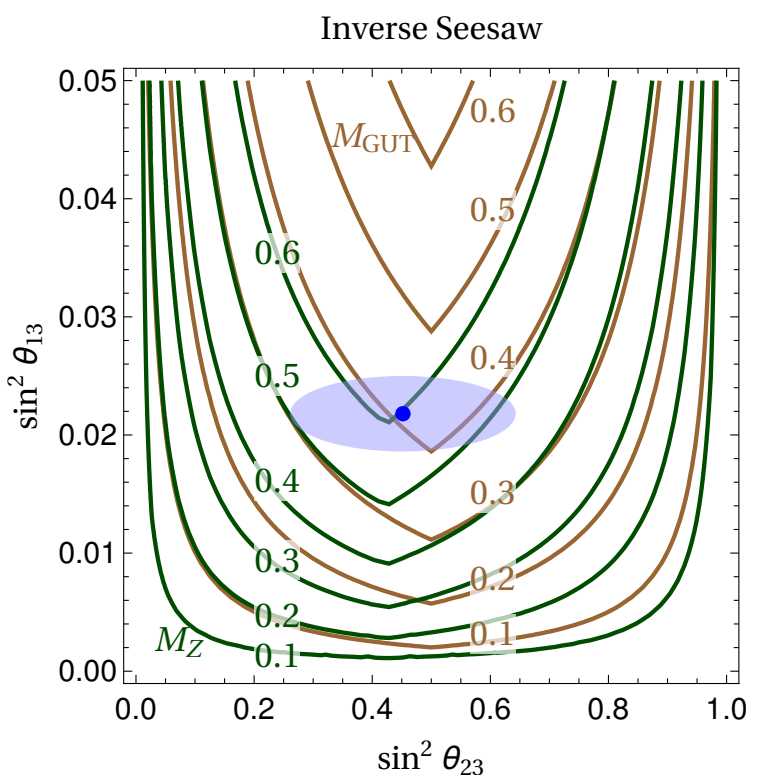

(b)

Figure 9. (a) Contours of equal probability $P_{\mathrm{KS}, \mathrm{NH}}$ (see eqs. (7) and (9) in the $\sin ^{2} \theta_{12}-\sin ^{2} \theta_{23}$ plane with RG effects included (dark green) and without RG effects (brown,see fig. 1) for the inverse seesaw model. We consider only parameter points with a normal mass ordering (see text in section III.1 for details). The blue ellipses denote the favored parameter region from the global fit in ref. [17] at the $3 \sigma$ confidence level, and the blue dot is the corresponding best fit point. We have fixed the third mixing angle at its best fit value, $\sin ^{2} \theta_{13}=0.0218$. With $R G$ effects included, the likelihood of the data with respect to the anarchy hypothesis increases from $41.1 \%$ to $59.3 \%$. (b) Contours of equal probability $P_{\mathrm{KS}, \mathrm{NH}}$ in the $\sin ^{2} \theta_{23}-\sin ^{2} \theta_{13}$ plane with and without RG effects included (same coloring as in (a)). The solar mixing angle is fixed at its best fit value, $\sin ^{2} \theta_{12}=0.304$. 


\begin{tabular}{ccc}
\hline \hline field & Spin & $U(1)_{B-L}$ charge $Q_{B-L}$ \\
\hline$\chi$ & 0 & -1 \\
$N_{R}$ & $\frac{1}{2}$ & -1 \\
$S$ & $\frac{1}{2}$ & +2 \\
$S^{\prime}$ & $\frac{1}{2}$ & -2 \\
$Z_{\mu}^{\prime}$ & 1 & 0 \\
\hline \hline
\end{tabular}

Table I. The new field content of the inverse seesaw model with gauged $U(1)_{B-L}$. All listed fields are SM singlets. As usual, standard model leptons have $B-L$ charge -1 , while quarks have $B-L$ charges of $1 / 3$.

the anarchy hypothesis with the data substantially: $\tilde{P}_{\mathrm{KS}}$ increases from $41.1 \%$ without RG effects to $59.3 \%$ with RG running included.

\section{III.3. Inverse Seesaw model with gauged $U(1)_{B-L}$}

As an example for a specific realization of the inverse seesaw mechanism, we consider an extension of the SM where the difference of baryon number and lepton number, $B-L$, is gauged [41. The corresponding gauge boson $Z^{\prime}$ is assumed to have a mass of order $100 \mathrm{TeV}$ generated by the vacuum expectation value (vev) of a new scalar $\chi$ with quantum numbers $(1,1,0,-1)$ under $S U(3)_{c} \times S U(2)_{L} \times U(1)_{Y} \times U(1)_{B-L}$. The fermionic field content of the model is the same as in the phenomenological inverse seesaw model discussed in section III.2 $\left(n_{N}\right.$ singlets $N_{R}, n_{S}$ singlets $S$ ), but the fermions now carry $B-L$ charge: the $N_{R}$ have $B-L$ charge $Q_{B-L}=-1$, while the $S$ have $Q_{B-L}=+2$. To ensure anomaly cancellation, it is necessary to supplement the model by $n_{S}$ extra Weyl fermions $S^{\prime}$ with $B-L$ charge -211 For simplicity, we assume that couplings of $S^{\prime}$ to other fields (except the $U(1)_{B-L}$ gauge boson) are forbidden by a new discrete symmetry. We will here remain agnostic with regard to the details of this symmetry and simply ignore $S^{\prime}$ in our subsequent discussion. The quantum numbers of the new particles in the inverse seesaw model with gauged $B-L$ are summarized in table $\mathrm{T}$.

The Lagrangian reads 41 .

$$
\begin{aligned}
\mathcal{L} \supset-\frac{1}{4} Z_{\mu \nu}^{\prime} Z^{\prime \mu \nu}+\sum_{\psi=N_{R}, S, S^{\prime}} i \bar{\psi} \not D \psi+ & \left(D_{\mu} \chi\right)\left(D^{\mu} \chi\right)^{\dagger}-\left[\left(Y_{\nu}^{\dagger}\right)^{\alpha \beta} \bar{L}_{L}^{\alpha} \tilde{H} N_{R}^{\beta}+\lambda_{S}^{\alpha \beta} \bar{S}^{\alpha} \chi^{\dagger}\left(N_{R}^{\beta}\right)^{c}+h . c .\right] \\
& +\mu_{2} \chi^{\dagger} \chi+\lambda_{2}\left(\chi^{\dagger} \chi\right)\left(H^{\dagger} H\right)+\lambda_{3}\left(\chi^{\dagger} \chi\right)^{2}+\frac{1}{\Lambda^{3}} \overline{S^{c}} \chi^{4} S, \quad
\end{aligned}
$$

where $D_{\mu}=\partial_{\mu}-i g_{B-L} Q_{B-L} Z_{\mu}^{\prime}$ is the covariant derivative. Note that the last term in the second row is dimension 7 and is therefore suppressed by three powers of a cutoff scale $\Lambda .2^{2}$ We have not written down other higher-dimensional operators here since they are not relevant to the phenomenology we are interested in.

${ }^{1}$ The cancellation of the triangular gauge anomaly requires that $\sum_{\psi_{L}}\left(Q_{B-L}^{\psi_{L}}\right)^{3}=\sum_{\psi_{R}}\left(Q_{B-L}^{\psi_{R}}\right)^{3}$, where $\psi_{L}$ and $\psi_{R}$ represent left-handed and right-handed fields, respectively.

2 A realization of this term in a renormalizable model requires additional fields. As a proof of principle, we could add a Majorana fermion $N_{R}^{\prime}$ and a real scalar $\rho$, both of which are total singlets under the SM gauge group and under $U(1)_{B-L}$, plus a complex scalar $\eta$ with $B-L$ charge -2 . If $N_{R}^{\prime}, \rho$ and $\eta$ are charged under a new $\mathbb{Z}_{2}$ symmetry under which all other fields are even, the operator $\overline{S^{c}} \chi^{4} S / \Lambda^{3}$ is generated through the following diagram:

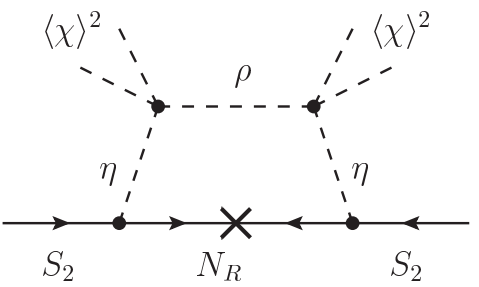




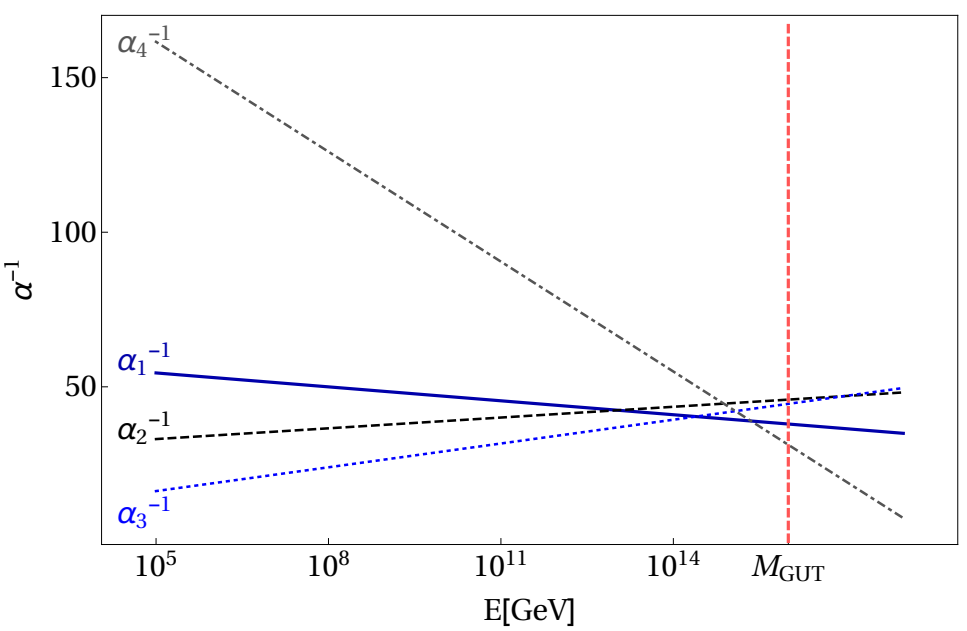

Figure 10. Running of the fine structure constant $\alpha_{1}$ for $U(1)_{Y}$ (dark blue solid), $\alpha_{2}$ for $S U(2)_{L}$ (black dashed), $\alpha_{3}$ for $S U(3)_{c}$ (blue dotted), and $\alpha_{4} \equiv g_{B-L}^{2} /(4 \pi)$ for $U(1)_{B-L}$ (dark gray dot-dashed) in the inverse seesaw model with gauged $B-L$.

As the vev of the new scalar $\chi$ breaks the $U(1)_{B-L}$ symmetry spontaneously, it endows the $B-L$ gauge boson with a mass $M_{Z^{\prime}}=\sqrt{2} g_{B-L}\langle\chi\rangle$. Moreover, it generates a Dirac-type mass mixing term of the form $\left(M_{D}\right)^{\alpha \beta} \bar{S}^{\alpha}\left(N_{R}^{\beta}\right)^{c}$ with $M_{D}=\lambda_{S}\left\langle\chi^{\dagger}\right\rangle$, and a Majorana mass term $m_{s} \overline{S^{c}} S$ with $m_{s}=\langle\chi\rangle^{4} / \Lambda^{3}$. Note that, in the unbroken phase of $U(1)_{B-L}$ a Majorana mass term for $S$ is forbidden due to its nonzero $B-L$ charge. After $U(1)_{B-L}$-breaking and electroweak symmetry breaking, the neutral components of the SM Higgs doublet $H$ and the new scalar $\chi$ mix to form two $C P$ even scalars [42].

Since our main goal in this section is only to explore whether a UV completion of the inverse seesaw model leads to qualitatively different RG effects, we make several simplifying assumptions in our RG analysis. First, to avoid having to deal with the multitude of different effective theories that can arise depending on where $B-L$ is broken relative to the masses of the heavy $\mathcal{O}(\mathrm{TeV})$ neutrinos, we always fix the $U(1)_{B-L}$ breaking scale at $f_{B-L}=100 \mathrm{TeV}$, well above the heavy neutrino masses. Moreover, we assume for simplicity that the $B-L$ breaking scale $f_{B-L}$, the vev $\langle\chi\rangle$, and the mass $m_{\chi}$ of $\chi$ are identical, so that we do not need to consider running between these scales. We choose the numerical value of $g_{B-L}$ such that it unifies to the extent possible at the GUT scale with the SM gauge couplings, see fig. 10. We find that a reasonable choice is $g_{B-L}=0.58$ at the GUT scale. By demanding that the running $Z^{\prime}$ mass parameter $M_{Z^{\prime}}(\mu) \equiv \sqrt{2} g_{B-L}(\mu)\langle\chi\rangle$ equals the scale $\mu$, we find the threshold mass $M_{Z^{\prime}} \approx 39 \mathrm{TeV}$. Since this is sufficiently close to $f_{B-L}=100 \mathrm{TeV}$ on a $\log$ scale, we integrate out $\chi$ and $Z^{\prime}$ simultaneously. As a further simplification, we ignore renormalization group effects on the quadratic terms in the scalar potential, and consequently the scale dependence of the scalar vevs. Since the scalar quartic couplings do not affect the flavor structure, their only influence on the neutrino mixing parameter can be indirect, by changing the various mass scales of the theory. Finally, we neglect kinetic mixing between the $Z^{\prime}$ and the standard model hypercharge gauge boson $B$, which would be allowed by the symmetries. Kinetic mixing is in general absent at the grand unification scale, where we assume that $U(1)_{B-L}$ emerges from a larger, non-Abelian gauge group. Even though kinetic mixing does get induced in the renormalization group evolution to lower scales at one loop level, its effect on other running parameters is suppressed by two loop factors and therefore negligible compared to other parameters appearing in the RG equations. In the same spirit we will also assume the Higgs portal coupling $\lambda_{2}$ to be strongly suppressed. 


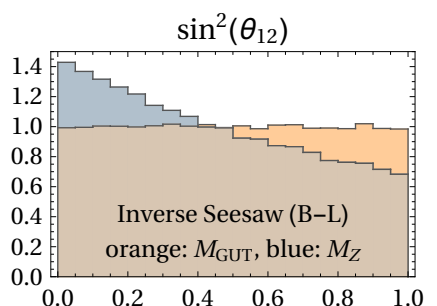

(a) Distribution of $\sin ^{2} \theta_{12}$

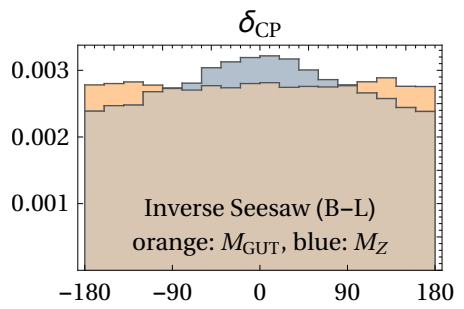

(d) Distribution of $\left.\delta_{\mathrm{CP}}{ }^{\circ}\right]$

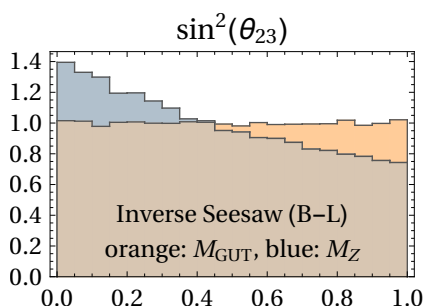

(b) Distribution of $\sin ^{2} \theta_{23}$

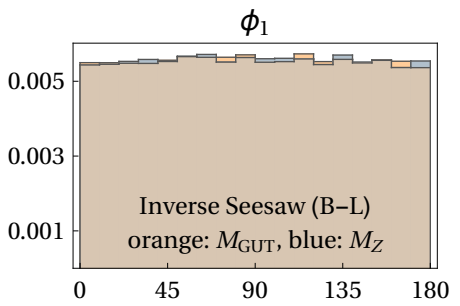

(e) Distribution of $\phi_{1}\left[^{\circ}\right]$

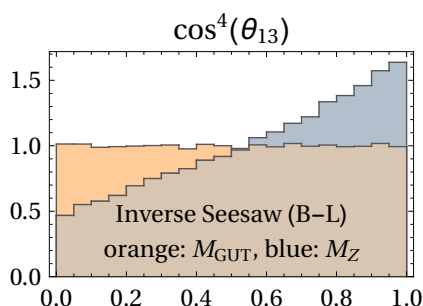

(c) Distribution of $\cos ^{4} \theta_{13}$

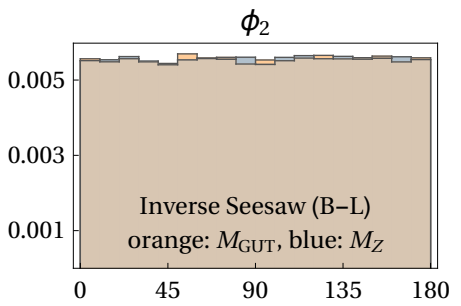

(f) Distribution of $\phi_{2}\left[^{\circ}\right]$

Figure 11. Distributions of the mixing angles and physical CP phases before and after renormalization group running in the inverse seesaw model with gauged $B-L$. The orange regions correspond to the parameters at $M_{\mathrm{GUT}}$, h scale, the blue regions to the parameters at $M_{Z}$. We find that the distributions are practically identical to those in the phenomenological inverse seesaw model from section [III.2, see fig. 7 ]

With these simplifications, the effective theory valid at scales $\mu<f_{B-L}$ is simply the one discussed in section III.2. We thus only need to evolve the parameters of the full model from $M_{\mathrm{GUT}}$ to $f_{B-L}$ and then feed them into the previously discussed evolution equations for the phenomenological inverse seesaw model from section III.2. The renormalization group equations for the inverse seesaw model with gauged $B-L$ are given in appendix $\mathrm{B}$. While their form is independent of the number of generations of singlet fields we introduce, we choose to invoke three $N_{R}$ fields and four $S$ fields, yielding one $\mathcal{O}(\mathrm{keV})$ mass-eigenstate as a possible DM candidate.

Proceeding as we did in sections III.1 and III.2, we investigate how the statistical distributions of the neutrino mixing parameters change due to RG effects in the inverse seesaw model with gauged $B-L$. We generate $10^{5}$ parameter sets at $M_{\mathrm{GUT}}$ and evolve them down to $M_{Z}$. In fig. 11 we show the distributions of the mixing parameters before and after running, and in fig. 12 we plot the distributions of the RG-induced shifts $\Delta x$. We find that these distributions are practically identical to those in the phenomenological inverse seesaw model without gauged $B-L$ number. Consequently, also the value of $\tilde{P}_{\mathrm{KS}}$ describing the compatibility of the data with anarchy is similar: $60.4 \%$, compared to $59.3 \%$ in the phenomenological model. The running of the light neutrino masses is somewhat larger than in the phenomenological model, though: the average anomalous dimensions is -0.051 , corresponding to a decrease of the mass eigenvalues by $80 \%$.

\section{SUMMARY AND CONCLUSIONS}

In summary, we have studied how renormalization group effects alter the predictions of neutrino anarchy. We have found that the mixing angles at $M_{Z}$ are on average several degrees smaller than at $M_{\mathrm{GUT}}$, and that the statistical distribution of the Dirac CP phase $\delta_{\mathrm{CP}}$ after RG running peaks at zero. The Kolmogorov-Smirnov $p$-value describing the compatibility of the observed mixing angles with the anarchy hypothesis thus increases from $41.1 \%$ without RG running to $47.7 \%$ with RG running in the type-I seesaw model, to $59.3 \%$ with RG running in the phenomenological inverse seesaw model, and to $60.4 \%$ with RG running in the inverse seesaw model with gauged $B-L$. 


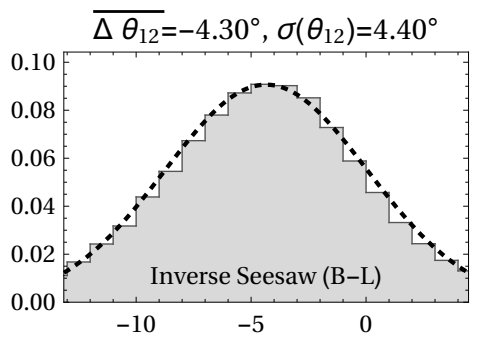

(a) Distribution of $\Delta \theta_{12}\left[^{\circ}\right]$

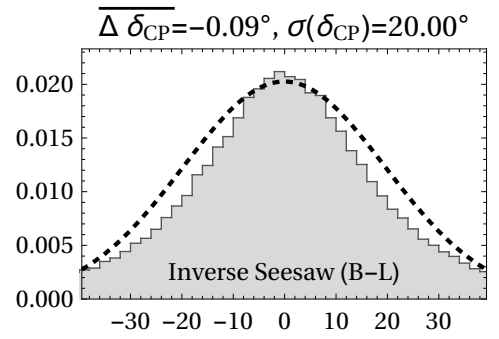

(d) Distribution of $\Delta \delta_{\mathrm{CP}}\left[^{\circ}\right]$

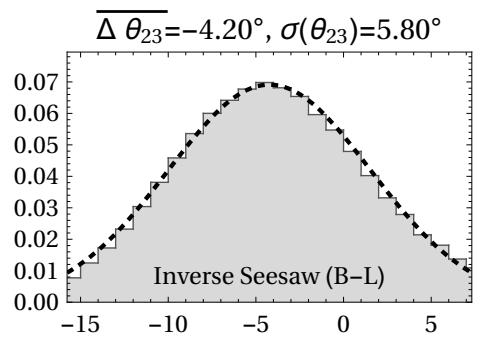

(b) Distribution of $\Delta \theta_{23}\left[^{\circ}\right]$

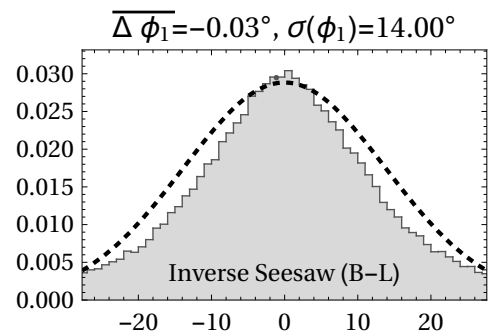

(e) Distribution of $\Delta \phi_{1}\left[^{\circ}\right]$

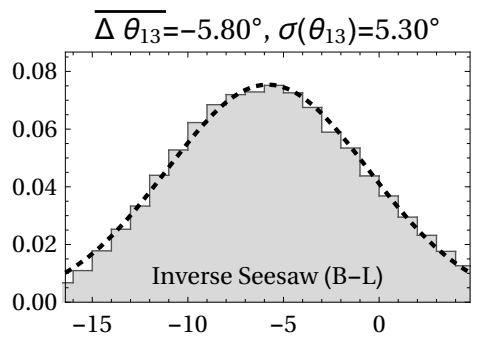

(c) Distribution of $\Delta \theta_{13}\left[^{\circ}\right]$

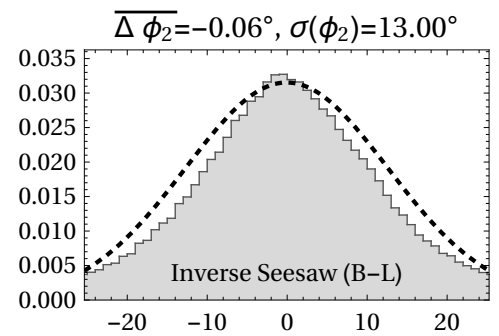

(f) Distribution of $\Delta \phi_{2}\left[^{\circ}\right]$

Figure 12. Shifts in the mixing angles and the CP phases during RG evolution form $M_{\mathrm{GUT}}$ to $M_{Z}$ in the inverse seesaw model with gauged $B-L$, obtained from $10^{5}$ randomly generated mass matrices. We use the notation $\Delta x \equiv x\left(M_{Z}\right)-x\left(M_{\mathrm{GUT}}\right)$, where $x$ stands for any of the mixing angles or complex phases. Also shown are Gaussian fits with central values $\overline{\Delta x}$ and widths $\sigma(\Delta x)$. The distributions are practically identical to those in the phenomenological inverse seesaw model from section III.2, see fig. 8 .

Our results thus strengthen the claim that anarchy offers a good description of neutrino oscillation data, and they demonstrate that RG effects are crucial and need to be taken into account when deriving quantitative predictions in the anarchy scenario.

\section{ACKNOWLEDGMENTS}

It is a pleasure to thank André de Gouvea, Jörn Kersten, and Hitoshi Murayama for useful discussions. We are moreover grateful to Alexander Merle for help in generating fig. 6, based on his papers [43, 44]. The work of VB and JK is supported by the German Research Foundation (DFG) under Grant Nos. KO 4820/1-1 and FOR 2239 and by the European Research Council (ERC) under the European Union's Horizon 2020 research and innovation programme (grant agreement No. 637506, " $\nu$ Directions"). VB and MK are supported by the DFG Graduate School Symmetry Breaking in Fundamental Interactions (GRK 1581). Additional support has been provided by the Cluster of Excellence "Precision Physics, Fundamental Interactions and Structure of Matter" (PRISMA - EXC 1098), grant No. 05H12UME of the German Federal Ministry for Education and Research (BMBF).

\section{Appendix A: Renormalization group equations for the type-I seesaw and inverse seesaw models}

In this appendix, we list the renormalization group equations for the inverse seesaw model discussed in section III.2. By simply setting certain terms to zero in these equations, also the RG equations for the type-I seesaw will follow (see appendix 3 . 


\section{Structure of the mass matrix}

We start from the mass matrix of the inverse seesaw model, eq. (18), which we repeat here:

$$
M_{\nu} \equiv\left(\begin{array}{ccc}
0 & Y_{\nu}^{T} v / \sqrt{2} & 0 \\
Y_{\nu} v / \sqrt{2} & 0 & M_{D}^{T} \\
0 & M_{D} & m_{s}
\end{array}\right)
$$

For 3 generations of active neutrinos, $n_{N}$ generations of heavy $N_{R}$ fields, and $n_{S}$ generations of $S$ fields, $Y_{\nu}$ is an $n_{N} \times 3$ matrix, $M_{D}$ is an $n_{S} \times n_{N}$ matrix, and $m_{s}$ is an $n_{S} \times n_{S}$ matrix. We assume $\left\|Y_{\nu}\right\| \sim \mathcal{O}(1),\left\|M_{D}\right\| \sim \mathrm{TeV}$ and $\left\|m_{s}\right\| \sim \mathrm{keV}$. We moreover assume $n_{S}>n_{N}$ so that $M_{\nu}$ has at least one $\mathcal{O}(\mathrm{keV})$ eigenvalue, providing a dark matter candidate.

For the RG analysis, it is more convenient to work with a matrix which has diagonal elements much larger than the off-diagonal ones. Moreover, it is convenient to group the elements of this matrix by their typical scale. We therefore apply a unitary transformation $V$ to $M_{\nu}$ to bring it to the form

$$
\tilde{M}_{\nu} \equiv V^{T} M_{\nu} V=\left(\begin{array}{ccc}
0 & \hat{Y}_{\nu}^{T} v / \sqrt{2} & \tilde{Y}_{\nu}^{T} v / \sqrt{2} \\
\hat{Y}_{\nu} v / \sqrt{2} & M_{N} & m_{D}^{T} \\
\tilde{Y}_{\nu} v / \sqrt{2} & m_{D} & \hat{m}_{s}
\end{array}\right)
$$

$V$ is chosen such that $\left\|M_{N}\right\| \gg\left\|m_{D}\right\|,\left\|\hat{m}_{s} \sim \mathrm{keV}\right\|$ and $\left\|\hat{Y}_{\nu}\right\| \sim\left\|Y_{\nu}\right\| \gg\left\|\tilde{Y}_{\nu}\right\|$. Thus, $M_{N}$ is a $2 n_{N} \times 2 n_{N}$ matrix with eigenvalues of order $\mathrm{TeV}$ and $\hat{m}_{s}$ is an $\left(n_{S}-n_{N}\right) \times\left(n_{S}-n_{N}\right)$ matrix.

To construct $V$ explicitly, we first consider the unitary $n_{S} \times n_{S}$ matrix $u_{L}$ and the unitary $n_{N} \times n_{N}$ matrix $u_{R}$ which diagonalize $M_{D}$ according to

$$
M_{D}^{\text {diag }}=u_{L}^{\dagger} M_{D} u_{R}
$$

Here $M_{D}^{\text {diag }}$ means the diagonal matrix of eigenvalues, ordered in descending order. From $u_{L}$ and $u_{R}$, we construct

$$
U_{L}=\left(\begin{array}{cc}
\mathbf{1}_{3+n_{N}} & 0 \\
0 & u_{L}
\end{array}\right) \quad U_{R}=\left(\begin{array}{ccc}
\mathbf{1}_{3} & 0 & 0 \\
0 & u_{R} & 0 \\
0 & 0 & \mathbf{1}_{n_{S}}
\end{array}\right) \quad W=U_{R} U_{L}^{*}
$$

Then, the transformation

$$
W^{T} M_{\nu} W
$$

will diagonalize the $M_{D}$ blocks in $m_{\nu}$, putting the largest mass eigenvalues on top. After this, we perform a rotation by $\pi / 4$ in the singlet block, given by:

$$
X=\left(\begin{array}{ccc}
\mathbf{1}_{3} & 0 & 0 \\
0 & r_{n_{N} \times n_{N}} & r_{n_{N} \times n_{S}} \\
0 & -r_{n_{S} \times n_{N}} & r_{n_{S} \times n_{S}}
\end{array}\right),
$$

where

$$
r_{n \times m} \equiv \frac{1}{\sqrt{2}}\left(\begin{array}{cccccc}
1 & \cdots & 0 & 0 & \cdots & 0 \\
\vdots & \ddots & \vdots & \vdots & \ddots & \vdots \\
0 & \cdots & 1 & 0 & \cdots & 0
\end{array}\right)
$$




\begin{tabular}{|c|c|c|c|c|}
\hline \multicolumn{2}{|c|}{ Original basis } & \multicolumn{3}{|c|}{ Rotated basis } \\
\hline matrix & dimensions & matrix & dimensions & scale \\
\hline$Y_{\nu}$ & $n_{N} \times 3$ & $\hat{Y}_{\nu}$ & $2 n_{N} \times 3$ & $\mathcal{O}\left(Y_{\nu}\right)$ \\
\hline$M_{D}$ & $n_{S} \times n_{N}$ & $\tilde{Y}_{\nu}$ & $\left(n_{S}-n_{N}\right) \times 3$ & $\mathcal{O}\left(Y_{\nu} m_{s} / M_{D}\right)$ \\
\hline \multirow[t]{3}{*}{$m_{s}$} & $n_{S} \times n_{S}$ & $M_{N}$ & $2 n_{N} \times 2 n_{N}$ & $\mathcal{O}\left(M_{D}\right)$ \\
\hline & & $m_{D}$ & $\left(n_{S}-n_{N}\right) \times 2 n_{N}$ & $\mathcal{O}\left(m_{s}\right)$ \\
\hline & & $\hat{m}_{s}$ & $\left(n_{S}-n_{N}\right) \times\left(n_{S}-n_{N}\right)$ & $\mathcal{O}\left(m_{s}\right)$ \\
\hline
\end{tabular}

Table II. Dimensions of the submatrices forming the mass matrices $M_{\nu}$ and $\tilde{M}_{\nu}$ in the inverse seesaw model (see eqs. A1) and (A2)). In the last column, we indicate the typical mass scale of the matrices in the rotated basis, eq. (A2).

is the $n \times n$ identity matrix (multiplied by $1 / \sqrt{2}$ ), padded with zeroes to turn it into an $n \times m$ matrix. After applying the full rotation

$$
V \equiv U_{R} U_{L}^{*} X
$$

to $M_{\nu}$, we obtain $\tilde{M}_{\nu}$ in the form given in eq. A2). We summarize the dimensions and the mass scales for the different blocks of $M_{\nu}$ and $\tilde{M}_{\nu}$ in table II. The $2 n_{N} \times 2 n_{N}$ matrix $M_{N}$ has $n_{N}$ pairs of eigenvalues that differ only by $\Delta M \sim \mathcal{O}(\mathrm{keV})$, much less than the absolute scale of the eigenvalues of order $\mathrm{TeV}$. The eigenstates in each pair couple to the active neutrinos via Yukawa couplings that are equal (up to a factor of $i$ ). Their contributions to the light neutrino masses therefore cancel up to terms of order $\Delta M /\left\|M_{N}\right\|$. This explains the smallness of the active neutrino masses despite the fact that $\hat{Y}_{\nu}$ is $\mathcal{O}(1)$ and $M_{N}$ is $\mathcal{O}(\mathrm{TeV})$. Another small contribution to the active neutrino masses comes from the $\mathcal{O}(\mathrm{keV})$ mass eigenstate mixing with the active fields through the strongly suppressed $\tilde{Y}_{\nu}$. We can also understand why $M_{\nu}$ has $n_{S}-n_{N}$ eigenvalues of order keV: the off-diagonal elements in the last $n_{S}-n_{N}$ rows and columns of $X$ are zero and thus we get $n_{S}-n_{N} \mathrm{keV}$ states that do not mix with the $N_{R}$ or $\nu_{L}$ fields.

\section{Beta functions for the inverse seesaw model}

We derive the RG equation for the mass matrix $\tilde{M}_{\nu}$ given in eq. A2 by using the fact that $\tilde{M}_{\nu}$ has exactly the same structure as the mass matrix of a type-I seesaw model.

Whenever the renormalization scale $\mu$ crosses the mass scale of one of the heavy sterile neutrinos as it is evolved from $M_{\mathrm{GUT}}$ to $M_{Z}$, the corresponding row and column is removed from the mass matrix $\tilde{M}_{\nu}$. Moreover the remaining rows and columns are modified to include the effect of integrating out one neutrino state. This in particular implies that the upper $3 \times 3$ block of $\tilde{M}_{\nu}$, which is zero initially, becomes a nonzero matrix $\kappa$. (We follow the notation of ref. [21] here.) If we did run down to sub-keV scales and integrated out all sterile states, $\kappa$ would eventually coincide with $m_{\nu}=\frac{v^{2}}{2} Y_{\nu}^{T} M_{D}^{-1} m_{s} M_{D}^{-1} Y_{\nu}$.

For the $\beta$ functions of a parameter $x$, we use the convention

$$
\beta_{x}=\mu \frac{d}{d \mu} x .
$$


The evolution equations read

$$
\begin{aligned}
& 16 \pi^{2} \beta_{M_{N}}=\left(\hat{Y}_{\nu} \hat{Y}_{\nu}^{\dagger}\right) M_{N}+M_{N}\left(\hat{Y}_{\nu} \hat{Y}_{\nu}^{\dagger}\right)^{T}+\left(\hat{Y}_{\nu} \tilde{Y}_{\nu}^{\dagger}\right) m_{D}+m_{D}^{T}\left(\hat{Y}_{\nu} \tilde{Y}_{\nu}^{\dagger}\right)^{T} \\
& 16 \pi^{2} \beta_{\hat{m}_{s}}=\left(\tilde{Y}_{\nu} \tilde{Y}_{\nu}^{\dagger}\right) \hat{m}_{s}+\hat{m}_{s}\left(\tilde{Y}_{\nu} \tilde{Y}_{\nu}^{\dagger}\right)^{T}+\left(\tilde{Y}_{\nu} \hat{Y}_{\nu}^{\dagger}\right) m_{D}^{T}+m_{D}\left(\tilde{Y}_{\nu} \hat{Y}_{\nu}^{\dagger}\right)^{T} \\
& 16 \pi^{2} \beta_{m_{D}}=\left(\tilde{Y}_{\nu} \tilde{Y}_{\nu}^{\dagger}\right) m_{D}+m_{D}\left(\hat{Y}_{\nu} \hat{Y}_{\nu}^{\dagger}\right)^{T}+\left(\tilde{Y}_{\nu} \hat{Y}_{\nu}^{\dagger}\right) M+\hat{m}_{s}\left(\hat{Y}_{\nu} \tilde{Y}_{\nu}^{\dagger}\right)^{T} \\
& 16 \pi^{2} \beta_{\kappa}=-\frac{3}{2}\left(Y_{e}^{\dagger} Y_{e}\right)^{T} \kappa-\frac{3}{2} \kappa\left(Y_{e}^{\dagger} Y_{e}\right)+\frac{1}{2}\left(\hat{Y}_{\nu}^{\dagger} \hat{Y}_{\nu}\right)^{T} \kappa+\frac{1}{2} \kappa\left(\hat{Y}_{\nu}^{\dagger} \hat{Y}_{\nu}\right) \\
& +2 \operatorname{tr}\left(Y_{e}^{\dagger} Y_{e}\right) \kappa+2 \operatorname{tr}\left(Y_{\nu}^{\dagger} Y_{\nu}\right) \kappa+6 \operatorname{tr}\left(Y_{u}^{\dagger} Y_{u}\right) \kappa \\
& +6 \operatorname{tr}\left(Y_{d}^{\dagger} Y_{d}\right) \kappa-3 g_{2}^{2} \kappa+\lambda \kappa \\
& 16 \pi^{2} \beta_{\hat{Y}_{\nu}}=\hat{Y}_{\nu}\left[\frac{3}{2}\left(\tilde{Y}_{\nu}^{\dagger} \tilde{Y}_{\nu}+\hat{Y}_{\nu}^{\dagger} \hat{Y}_{\nu}\right)-\frac{3}{2}\left(Y_{e}^{\dagger} Y_{e}\right)+\operatorname{tr}\left(\hat{Y}_{\nu}^{\dagger} \hat{Y}_{\nu}+\tilde{Y}_{\nu}^{\dagger} \tilde{Y}_{\nu}\right)\right. \\
& \left.+\operatorname{tr}\left(Y_{e}^{\dagger} Y_{e}\right)+3 \operatorname{tr}\left(Y_{u}^{\dagger} Y_{u}\right)+3 \operatorname{tr}\left(Y_{d}^{\dagger} Y_{d}\right)-\frac{9}{20} g_{1}^{2}-\frac{9}{4} g_{2}^{2}\right] \\
& 16 \pi^{2} \beta_{\tilde{Y}_{\nu}}=\tilde{Y}_{\nu}\left[\frac{3}{2}\left(\tilde{Y}_{\nu}^{\dagger} \tilde{Y}_{\nu}+\hat{Y}_{\nu}^{\dagger} \hat{Y}_{\nu}\right)-\frac{3}{2}\left(Y_{e}^{\dagger} Y_{e}\right)+\operatorname{tr}\left(\hat{Y}_{\nu}^{\dagger} \hat{Y}_{\nu}+\tilde{Y}_{\nu}^{\dagger} \tilde{Y}_{\nu}\right)\right. \\
& \left.+\operatorname{tr}\left(Y_{e}^{\dagger} Y_{e}\right)+3 \operatorname{tr}\left(Y_{u}^{\dagger} Y_{u}\right)+3 \operatorname{tr}\left(Y_{d}^{\dagger} Y_{d}\right)-\frac{9}{20} g_{1}^{2}-\frac{9}{4} g_{2}^{2}\right] \\
& 16 \pi^{2} \beta_{Y_{e}}=Y_{e}\left[\frac{3}{2} Y_{e}^{\dagger} Y_{e}-\frac{3}{2}\left(\hat{Y}_{\nu}^{\dagger} \hat{Y}_{\nu}+\tilde{Y}_{\nu}^{\dagger} \tilde{Y}_{\nu}\right)-\frac{9}{4} g_{1}^{2}-\frac{9}{4} g_{2}^{2}\right. \\
& \left.+\operatorname{tr}\left[Y_{e}^{\dagger} Y_{e}+\hat{Y}_{\nu}^{\dagger} \hat{Y}_{\nu}+\tilde{Y}_{\nu}^{\dagger} \tilde{Y}_{\nu}+3 Y_{d}^{\dagger} Y_{d}+3 Y_{u}^{\dagger} Y_{u}\right]\right] \\
& 16 \pi^{2} \beta_{Y_{d}}=Y_{d}\left[\frac{3}{2} Y_{d}^{\dagger} Y_{d}-\frac{3}{2} Y_{u}^{\dagger} Y_{u}-\frac{1}{4} g_{1}^{2}-\frac{9}{4} g_{2}^{2}-8 g_{3}^{2}\right. \\
& \left.+\operatorname{tr}\left[Y_{e}^{\dagger} Y_{e}+\hat{Y}_{\nu}^{\dagger} \hat{Y}_{\nu}+\tilde{Y}_{\nu}^{\dagger} \tilde{Y}_{\nu}+3 Y_{d}^{\dagger} Y_{d}+3 Y_{u}^{\dagger} Y_{u}\right]\right] \\
& 16 \pi^{2} \beta_{Y_{u}}=Y_{u}\left[\frac{3}{2} Y_{u}^{\dagger} Y_{u}-\frac{3}{2} Y_{d}^{\dagger} Y_{d}-\frac{17}{20} g_{1}^{2}-\frac{9}{4} g_{2}^{2}-8 g_{3}^{2}\right. \\
& \left.+\operatorname{tr}\left[Y_{e}^{\dagger} Y_{e}+\hat{Y}_{\nu}^{\dagger} \hat{Y}_{\nu}+\tilde{Y}_{\nu}^{\dagger} \tilde{Y}_{\nu}+3 Y_{d}^{\dagger} Y_{d}+3 Y_{u}^{\dagger} Y_{u}\right]\right] \\
& 16 \pi^{2} \beta_{\lambda}=6 \lambda^{2}-3 \lambda\left(3 g_{2}^{2}+\frac{3}{5} g_{1}^{2}\right)+3 g_{2}^{4}+\frac{3}{2}\left(\frac{3}{5} g_{1}^{2}+g_{2}^{2}\right)^{2} \\
& +4 \lambda \operatorname{tr}\left[Y_{e}^{\dagger} Y_{e}+\hat{Y}_{\nu}^{\dagger} \hat{Y}_{\nu}+\tilde{Y}_{\nu}^{\dagger} \tilde{Y}_{\nu}+3 Y_{d}^{\dagger} Y_{d}+3 Y_{u}^{\dagger} Y_{u}\right] \\
& -8 \operatorname{tr}\left[Y_{e}^{\dagger} Y_{e} Y_{e}^{\dagger} Y_{e}+\left(\hat{Y}_{\nu}^{\dagger} \hat{Y}_{\nu}+\tilde{Y}_{\nu}^{\dagger} \tilde{Y}_{\nu}\right)\left(\hat{Y}_{\nu}^{\dagger} \hat{Y}_{\nu}+\tilde{Y}_{\nu}^{\dagger} \tilde{Y}_{\nu}\right)\right. \\
& \left.+3 Y_{d}^{\dagger} Y_{d} Y_{d}^{\dagger} Y_{d}+3 Y_{u}^{\dagger} Y_{u} Y_{u}^{\dagger} Y_{u}\right]
\end{aligned}
$$

Note that the new fields introduced in the inverse seesaw model are all singlets under the SM gauge group. Therefore, the one-loop running of the $S U(3)_{c} \times S U(2)_{L} \times U(1)_{Y}$ gauge couplings is described by the SM expressions

$$
\begin{aligned}
& g_{1}^{-2}(\mu)=g_{1}^{-2}\left(M_{Z}\right)-\frac{41}{80 \pi^{2}} \log \left(\frac{\mu}{M_{Z}}\right), \\
& g_{2}^{-2}(\mu)=g_{2}^{-2}\left(M_{Z}\right)+\frac{19}{48 \pi^{2}} \log \left(\frac{\mu}{M_{Z}}\right), \\
& g_{3}^{-2}(\mu)=g_{3}^{-2}\left(M_{Z}\right)+\frac{7}{8 \pi^{2}} \log \left(\frac{\mu}{M_{Z}}\right) .
\end{aligned}
$$




\section{Beta functions for the type-I seesaw model}

By comparing the mass matrix of the inverse seesaw model, eq. A2, to the mass matrix of the type-I seesaw model

$$
M_{\nu, \text { type-I }} \equiv\left(\begin{array}{cc}
0 & Y_{\nu}^{T} v / \sqrt{2} \\
Y_{\nu} v / \sqrt{2} & M
\end{array}\right)
$$

we can immediately read off that the RGEs for the type-I seesaw model are obtained from eqs. A10 to A22 by setting $\hat{Y}_{\nu}=Y_{\nu}, M_{N}=M, \tilde{Y}_{\nu}=0, m_{D}=0$, and $\hat{m}_{s}=0$.

\section{Appendix B: Beta functions in the inverse seesaw model with gauged $B-L$}

For the model with the gauged $U(1)_{B-L}$ symmetry, described in section III.3, we find the following evolution equations:

$$
\begin{aligned}
16 \pi^{2} \beta_{Y_{\nu}}=Y_{\nu}\left[\frac{1}{2} \lambda_{S}^{T} \lambda_{S}^{*}\right]+Y_{\nu}\left[\frac{3}{2} Y_{\nu}^{\dagger} Y_{\nu}-\frac{3}{2} Y_{e}^{\dagger} Y_{e}+\operatorname{tr}\left(Y_{e}^{\dagger} Y_{e}\right)+\operatorname{tr}\left(Y_{\nu}^{\dagger} Y_{\nu}\right)\right. & \left.+3 \operatorname{tr}\left(Y_{d}^{\dagger} Y_{d}\right)+3 \operatorname{tr}\left(Y_{u}^{\dagger} Y_{u}\right)-\frac{9}{20} g_{1}^{2}-\frac{9}{4} g_{2}^{2}-6 g^{\prime 2}\right] \\
16 \pi^{2} \beta_{Y_{e}}=Y_{e}[ & {\left[\frac{3}{2} Y_{e}^{\dagger} Y_{e}-\frac{3}{2} Y_{\nu}^{\dagger} Y_{\nu}+\operatorname{tr}\left(Y_{e}^{\dagger} Y_{e}\right)+\operatorname{tr}\left(Y_{\nu}^{\dagger} Y_{\nu}\right)+3 \operatorname{tr}\left(Y_{d}^{\dagger} Y_{d}\right)\right.} \\
& \left.+3 \operatorname{tr}\left(Y_{u}^{\dagger} Y_{u}\right)-\frac{9}{4} g_{1}^{2}-\frac{9}{4} g_{2}^{2}-6 g^{\prime 2}\right] \\
16 \pi^{2} \beta_{\lambda_{S}}=\lambda_{S} & {\left[\operatorname{tr}\left(\lambda_{S}^{\dagger} \lambda_{S}\right)+Y_{\nu}^{*} Y_{\nu}^{T}+\lambda_{S}^{\dagger} \lambda_{S}-15 g^{\prime 2}\right] } \\
16 \pi^{2} \beta_{Y_{u}}=Y_{u} & {\left[\frac{3}{2} Y_{u}^{\dagger} Y_{u}-\frac{3}{2} Y_{d}^{\dagger} Y_{d}-\frac{17}{20} g_{1}^{2}-\frac{9}{4} g_{2}^{2}-8 g_{3}^{2}-\frac{2}{3} g^{\prime 2}\right.} \\
& \left.+\operatorname{tr}\left(Y_{e}^{\dagger} Y_{e}\right)+\operatorname{tr}\left(Y_{\nu}^{\dagger} Y_{\nu}\right)+3 \operatorname{tr}\left(Y_{d}^{\dagger} Y_{d}\right)+3 \operatorname{tr}\left(Y_{u}^{\dagger} Y_{u}\right)\right] \\
& {\left[3 \frac{3}{2} Y_{d}^{\dagger} Y_{d}-\frac{3}{2} Y_{u}^{\dagger} Y_{u}-\frac{1}{4} g_{1}^{2}-\frac{9}{4} g_{2}^{2}-8 g_{3}^{2}-\frac{2}{3} g^{\prime 2}\right.} \\
& \left.+\operatorname{tr}\left(Y_{e}^{\dagger} Y_{e}\right)+\operatorname{tr}\left(Y_{\nu}^{\dagger} Y_{\nu}\right)+3 \operatorname{tr}\left(Y_{d}^{\dagger} Y_{d}\right)+3 \operatorname{tr}\left(Y_{u}^{\dagger} Y_{u}\right)\right] \\
\left.16 \pi^{2} \beta_{Y_{d}}=Y_{d}\right) & g^{\prime 3} \\
16 \pi^{2} \beta_{g^{\prime}}= &
\end{aligned}
$$

The numerical coefficient $C$ in the beta function for the $U(1)_{B-L}$ coupling constant $g^{\prime}$ in the last line, eq. (B6), depends on the number of fields in the model and is given by

$$
C=\frac{2}{3} \sum_{\text {fermions }} Q_{B-L}^{2}+\frac{1}{3} \sum_{\text {scalars }} Q_{B-L}^{2}
$$

In our specific model, including four generations of $S$ and $S^{\prime}$ each, we find $C=\frac{97}{3}$. For only three generations of $S$ and $S^{\prime}$ fields, we would have found $C=27$ instead. The running of the quartic Higgs coupling $\lambda$ and of the SM gauge couplings is the same as in the phenomenological inverse seesaw model at one-loop level, see previous section. Note that we neglect the running 
of all parameters in the scalar sector other than the quartic Higgs coupling. The running of these couplings influences the evolution of the mixing parameters only indirectly by modifying the $U(1)_{B-L}$ breaking scale.

Where possible, we have checked that we reproduce the applicable terms in the renormalization group equations presented in ref. [45] for a $U(1)_{B-L}$ extension of the SM with three right-handed neutrinos, but without an inverse seesaw mechanism.

[1] L. J. Hall, H. Murayama, and N. Weiner, Neutrino mass anarchy, Phys.Rev.Lett. 84 (2000) $2572-2575$, hep-ph/9911341.

[2] N. Haba and H. Murayama, Anarchy and hierarchy, Phys.Rev. D63 (2001) 053010, hep-ph/0009174.

[3] A. de Gouvea and H. Murayama, Statistical test of anarchy, Phys.Lett. B573 (2003) 94-100, hep-ph/0301050.

[4] J. Espinosa, Anarchy in the neutrino sector?, hep-ph/0306019.

[5] A. de Gouvea and H. Murayama, Neutrino Mixing Anarchy: Alive and Kicking, 1204.1249.

[6] J. Heeck and W. Rodejohann, Sterile neutrino anarchy, Phys. Rev. D87 (2013), no. 3 037301, 1211.5295 .

[7] J. Gluza and R. Szafron, Real and complex random neutrino mass matrices and theta13, Phys.Rev. D85 (2012) 047701, 1111.7278. v3: Discussion about 3+1 scenario extended, fig 5,6 added.

[8] K. Agashe, T. Okui, and R. Sundrum, A Common Origin for Neutrino Anarchy and Charged Hierarchies, Phys.Rev.Lett. 102 (2009) 101801, 0810.1277.

[9] G. Altarelli, F. Feruglio, I. Masina, and L. Merlo, Repressing Anarchy in Neutrino Mass Textures, JHEP 1211 (2012) 139, 1207.0587.

[10] G. Altarelli, F. Feruglio, and I. Masina, Models of neutrino masses: Anarchy versus hierarchy, JHEP 0301 (2003) 035, hep-ph/0210342.

[11] D. L. Bennett, H. B. Nielsen, and I. Picek, Understanding Fine Structure Constants and Three Generations, Phys. Lett. B208 (1988) 275.

[12] J. F. Donoghue, K. Dutta, and A. Ross, Quark and lepton masses and mixing in the landscape, Phys. Rev. D73 (2006) 113002, hep-ph/0511219.

[13] J. F. Donoghue, K. Dutta, A. Ross, and M. Tegmark, Likely values of the Higgs vev, Phys. Rev. D81 (2010) 073003, 0903.1024.

[14] S. Antusch, J. Kersten, M. Lindner, and M. Ratz, Running neutrino masses, mixings and CP phases: Analytical results and phenomenological consequences, Nucl. Phys. B674 (2003) 401-433, hep-ph/0305273.

[15] A. de Gouvea and J. Jenkins, The Physical Range of Majorana Neutrino Mixing Parameters, Phys.Rev. D78 (2008) 053003, 0804.3627.

[16] X. Lu and H. Murayama, Neutrino Mass Anarchy and the Universe, JHEP 1408 (2014) 101, 1405.0547.

[17] M. Gonzalez-Garcia, M. Maltoni, and T. Schwetz, Updated fit to three neutrino mixing: status of leptonic CP violation, JHEP 1411 (2014) 052, 1409.5439.

[18] J. Bergstrom, D. Meloni, and L. Merlo, A Bayesian comparison of U(1) lepton flavour models, Phys.Rev. D89 (2014) 093021, 1403.4528.

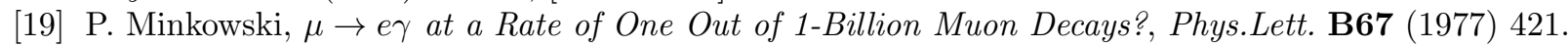

[20] R. N. Mohapatra and G. Senjanović, Neutrino mass and spontaneous parity nonconservation, Phys. Rev. Lett. 44 (Apr, 1980) 912-915.

[21] S. Antusch, J. Kersten, M. Lindner, M. Ratz, and M. A. Schmidt, Running neutrino mass parameters in see-saw scenarios, JHEP 0503 (2005) 024, hep-ph/0501272.

[22] J.-w. Mei, Running neutrino masses, leptonic mixing angles and CP-violating phases: From M(Z) to Lambda(GUT), Phys.Rev. D71 (2005) 073012, hep-ph/0502015.

[23] J. Casas, J. Espinosa, A. Ibarra, and I. Navarro, General RG equations for physical neutrino parameters and their phenomenological implications, Nucl.Phys. B573 (2000) 652-684, hep-ph/9910420.

[24] P. H. Chankowski and Z. Pluciennik, Renormalization group equations for seesaw neutrino masses, 
Phys.Lett. B316 (1993) 312-317, hep-ph/9306333.

[25] T. Ohlsson and S. Zhou, Renormalization group running of neutrino parameters, Nature Commun. $\mathbf{5}$ (2014) 5153, 1311.3846.

[26] N. Haba, K. Kaneta, R. Takahashi, and Y. Yamaguchi, Accurate renormalization group analyses in neutrino sector, Nucl.Phys. B885 (2014) 180-195, 1402.4126].

[27] M. A. Schmidt, Renormalization group evolution in the type I+ II seesaw model, Phys. Rev. D76 (2007) 073010, 0705.3841]. [Erratum: Phys. Rev.D85,099903(2012)].

[28] Planck Collaboration, P. Ade et al., Planck 2013 results. XVI. Cosmological parameters, Astron.Astrophys. 571 (2014) A16, 1303.5076.

[29] GERDA Collaboration, M. Agostini et al., Results on Neutrinoless Double- $\beta$ Decay of ${ }^{76}$ Ge from Phase I of the GERDA Experiment, Phys.Rev.Lett. 111 (2013), no. 12 122503, 1307.4720].

[30] EXO-200 Collaboration Collaboration, J. Albert et al., Search for Majorana neutrinos with the first two years of EXO-200 data, Nature $\mathbf{5 1 0}$ (2014) 229234, 1402.6956.

[31] Y. Huang and B.-Q. Ma, Constraints on absolute neutrino Majorana mass from current data, Universe 2 (2014), no. 3 65-71, 1407.4357].

[32] R. N. Mohapatra and J. W. F. Valle, Neutrino mass and baryon-number nonconservation in superstring models, Phys. Rev. D 34 (Sep, 1986) 1642-1645.

[33] R. N. Mohapatra, Mechanism for understanding small neutrino mass in superstring theories, Phys. Rev. Lett. 56 (Feb, 1986) 561-563.

[34] C. Carvajal, A. Dias, C. Nishi, and B. Snchez-Vega, Axion Like Particles and the Inverse Seesaw Mechanism, 1503.03502 .

[35] A. Dias, C. de S. Pires, P. Rodrigues da Silva, and A. Sampieri, A Simple Realization of the Inverse Seesaw Mechanism, Phys.Rev. D86 (2012) 035007, 1206.2590.

[36] A. Dias, C. de S. Pires, and P. R. da Silva, How the Inverse See-Saw Mechanism Can Reveal Itself Natural, Canonical and Independent of the Right-Handed Neutrino Mass, Phys.Rev. D84 (2011) 053011, 1107.0739 .

[37] P. Dev and R. Mohapatra, TeV Scale Inverse Seesaw in SO(10) and Leptonic Non-Unitarity Effects, Phys.Rev. D81 (2010) 013001, 0910.3924.

[38] A. Abada, G. Arcadi, and M. Lucente, Dark Matter in the minimal Inverse Seesaw mechanism, 1406.6556.

[39] P. B. Dev and A. Pilaftsis, Light and Superlight Sterile Neutrinos in the Minimal Radiative Inverse Seesaw Model, 1212.3808 .

[40] J. Bergstrom, M. Malinsky, T. Ohlsson, and H. Zhang, Renormalization group running of neutrino parameters in the inverse seesaw model, Phys.Rev. D81 (2010) 116006, 1004.4628.

[41] S. Khalil, TeV-scale gauged B-L symmetry with inverse seesaw mechanism, Phys.Rev. D82 (2010) 077702, 1004.0013 .

[42] W. Emam and S. Khalil, Higgs and Z-prime phenomenology in B-L extension of the standard model at LHC, Eur.Phys.J. C52 (2007) 625-633, 0704.1395.

[43] M. Lindner, A. Merle, and W. Rodejohann, Improved limit on theta(13) and implications for neutrino masses in neutrino-less double beta decay and cosmology, Phys.Rev. D73 (2006) 053005, hep-ph/0512143.

[44] A. Merle and W. Rodejohann, The Elements of the neutrino mass matrix: Allowed ranges and implications of texture zeros, Phys.Rev. D73 (2006) 073012, hep-ph/0603111.

[45] L. Basso, S. Moretti, and G. M. Pruna, A Renormalisation Group Equation Study of the Scalar Sector of the Minimal B-L Extension of the Standard Model, Phys.Rev. D82 (2010) 055018, 1004.3039. 